\title{
Hausdorff dimension and the Weil-Petersson extension to quasifuchsian space
}

\author{
MARTIN BRIDGEMAN
}

\begin{abstract}
We consider a natural nonnegative two-form $G$ on quasifuchsian space that extends the Weil-Petersson metric on Teichmüller space. We describe completely the positive definite locus of $G$, showing that it is a positive definite metric off the fuchsian diagonal of quasifuchsian space and is only zero on the "pure-bending" tangent vectors to the fuchsian diagonal. We show that $G$ is equal to the pullback of the pressure metric from dynamics. We use the properties of $G$ to prove that an an critical point of the Hausdorff dimension function on quasifuchsian space the Hessian of the Hausdorff dimension function must be positive definite on at least a halfdimensional subspace of the tangent space. In particular this implies that Hausdorff dimension has no local maxima on quasifuchsian space.
\end{abstract}

30F60, 30F40, 37D35

\section{Statement of results}

Let $S$ be a closed hyperbolic surface and $T(S)$ be the associated Teichmüller space. Then the Weil-Petersson metric $w$ is a Riemannian metric on $T(S)$. For simplicity, we normalize the Weil-Petersson metric to define the normalized Weil-Petersson metric

$$
g=\left(\frac{2}{3 \pi|\chi(S)|}\right) w .
$$

If $\mathrm{QF}(S)$ is the quasifuchsian space of $S$, then by Bers simultaneous uniformization, $\mathrm{QF}(S) \simeq T(S) \times T(\bar{S})$ where $\bar{S}$ has opposite orientation to $S$. This gives the natural diagonal embedding $\Delta: T(S) \rightarrow T(S) \times T(\bar{S}) \simeq \mathrm{QF}(S)$ given by $\Delta(X)=(X, \bar{X})$. We let $F(S)=\Delta(T(S))$ the diagonal in $\mathrm{QF}(S)$. Then $F(S)$ corresponds to the subspace of fuchsian elements of $\mathrm{QF}(S)$ and is called the fuchsian subspace. It is a smooth submanifold of $\mathrm{QF}(S)$ and we have the natural identification $T(S) \simeq F(S)$ via $\Delta$.

Quasifuchsian space $\mathrm{QF}(S)$ has a complex structure arising out of identifying the isometry group $\operatorname{Isom}_{+}\left(\mathbb{H}^{3}\right)$ with $\operatorname{PSL}(2, \mathbb{C})$ (see Marden [9]). This complex structure is given by a bundle map $J: T(\mathrm{QF}(S)) \rightarrow T(\mathrm{QF}(S))$ with $J$ a lift of the identity map 
on $\mathrm{QF}(S)$ and $J^{2}=-I$ where $I$ the identity map on $T(\mathrm{QF}(S))$. If $v \in T(\mathrm{QF}(S))$ we will write $J . v=J(v)$ for simplicity.

We define $h: \mathrm{QF}(S) \rightarrow \mathbb{R}$ by setting $h(X)$ equal to the Hausdorff dimension of the limit set of $X$. Then by Ruelle [14], $h$ is real-analytic. Also, associated with each $X \in \mathrm{QF}(S)$ is the real-analytic length function $L_{\mu_{X}}: \mathrm{QF}(S) \rightarrow \mathbb{R}$ of the unit Patterson-Sullivan geodesic current $\mu_{X}$ of $X$.

In [6], the author and Taylor showed that the function $\left(h L_{\mu_{X}}\right)$ on $\mathrm{QF}(S)$ is minimum at $X$. Using this we defined a natural nonnegative two-form $G$ on $\mathrm{QF}(S)$ given by taking the Hessian of $\left(h L_{\mu_{X}}\right)$ at $X$. Thus

$$
G_{X}=\left(h L_{\mu_{X}}\right)^{\prime \prime}(X) .
$$

We showed that $G$ extends the normalized Weil-Petersson metric $g$ on $F(S)$. Specifically:

Theorem 1.1 (Bridgeman-Taylor [6]) There exists a continuous nonnegative twoform $G$ on $\mathrm{QF}(S)$ such that for all $X \in F(S) \subseteq \mathrm{QF}(S)$

$$
\langle v, w\rangle_{G}=\langle v, w\rangle_{g} \text { for all } v, w \in T_{X}(F(S)) \subset T_{X}(\mathrm{QF}(S)) .
$$

In this paper we answer the question of whether $G$ is positive-definite on $\mathrm{QF}(S)$. The answer is that $G$ is "almost" a metric, in particular it is a metric off the fuchsian locus $F(S)$. The complete description of the positive-definite locus of $G$ is given by the following Theorem.

Main Theorem Let $v \in T_{X}(\mathrm{QF}(S)), v \neq 0$. Then $\|v\|_{G}=0$ if and only if

(1) $X \in F(S)$,

(2) $v=J . w$ where $w \in T_{X}(F(S))$.

Although we will not discuss this aspect further, the Main Theorem has a simple description in terms of the geometry of the associated deformations. If $X \in F(S) \subseteq$ $\mathrm{QF}(S)$ then the tangent space at $X$ decomposes into $T_{X}(\mathrm{QF}(S))=T_{X}(F(S)) \oplus$ $J\left(T_{X}(F(S))\right.$ ) (see Bonahon [2]). If $w \in T_{X}(F(S))$ then $w$ corresponds to deforming $X$ inside the fuchsian subspace $F(S)$. By the Earthquake Theorem, this deformation is given by shearing (or twisting) $X$ along a certain measured lamination $\beta$ (see Kerckhoff [7]). Thus the vectors in $T_{X}(F(S))$ are called pure shearing vectors. If $v \in J\left(T_{X}(F(S))\right)$ then $v=J . w$ where $w$ is a pure shearing vector with some corresponding measured lamination $\beta$. It can be shown that $v$ then corresponds to 
deforming the structure $X$ by bending along measured lamination $\beta$ (see Bonahon [2]). Thus the vectors in $J\left(T_{X}(F(S))\right)$ are called pure bending vectors.

Therefore in terms of deformations, the Main Theorem states that the degenerate vectors for $G$ are exactly the pure bending vectors at fuchsian representations.

The proof of the Main Theorem is via the conformal equivalence of the two-form $G$ with another two-form $W$ obtained by taking the pullback of the so-called pressure metric of thermodynamics. Then the proof of positive-definiteness reduces to showing that the pullback is only trivial for the above tangent vectors. This relation between $G$ and $W$ was suggested by McMullen in the paper [11].

Using the Main Theorem we study properties of the critical points of $h$. In particular if $h$ is critical at $X$ then the Hessian of $h$ at $X$ is a well-defined symmetric bilinear two-form. Thus the Hessian has a well-defined signature. Applying the Main Theorem we obtain:

Theorem 1.2 If $X \in \mathrm{QF}(S)$ is a critical point of $h: \mathrm{QF}(S) \rightarrow \mathbb{R}$ then the Hessian of $h$ at $X$ is positive definite on a subspace of $T_{X}(\mathrm{QF}(S))$ of dimension at least $6 g-6$. In particular $h$ has no local maxima in $\mathrm{QF}(S)$.

\subsection{Background}

In [6], the complex structure on $\mathrm{QF}(S)$ was used to define a metric $H$ on Teichmüller space. If $X \in T_{X}(F(S))$ the associated two-form at $X$ is given by

$$
\langle v, w\rangle_{H}=h^{\prime \prime}(X)(J . v, J . w)
$$

for $v, w \in T_{X}(F(S)) \subseteq T_{X}(\mathrm{QF}(S))$.

From the definition of $G$ and the fact that it is nonnegative, we obtain:

Theorem 1.3 (Bridgeman-Taylor [6]) If $X \in F(S)$ and $v \in T_{X}(F(S)) \subseteq T_{X}(\mathrm{QF}(S))$ then

$$
0 \leq\|J . v\|_{G}^{2}=\|v\|_{H}^{2}-\|v\|_{g}^{2}
$$

where $g$ is the normalized Weil-Petersson metric. Thus the two-form $H$ is a positive definite metric on $F(S)$ and satisfies

$$
\|v\|_{H} \geq\|v\|_{g} .
$$

In [11], McMullen showed that the Weil-Petersson metric was equivalent to the second derivative of various well-defined Hausdorff dimension functions at the fuchsian locus. In particular McMullen proved the following Theorem.

Theorem 1.4 (McMullen [11]) The metrics $H$ and $g$ are equal. 
The results of this paper arise out of combining the methods outlined in the paper [6] with those of the paper of McMullen [11] and applying them in the nonfuchsian case. We note that a consequence of the Main Theorem is that $\|J . v\|_{G}=0$ when $v \in T_{X}(F(S))$ which we will show gives a new proof of McMullen's result above (Theorem 1.4).

Acknowledgements This paper is an outgrowth of work done in collaboration with Edward Taylor who I would like to especially thank. I would also like to particularly thank Curt McMullen for his many helpful suggestions on this project and for the paper [11]. I would further like to thank Francis Bonahon, Dick Canary, Jeremy Kahn and Rich Schwartz for their helpful conversations. Finally I would like to thank the reviewer who made many helpful suggestions.

This research was supported in part by NSF grant DMS-0707116.

\section{Kleinian groups and geodesic currents}

\subsection{Kleinian groups}

Let Isom $+\left(\mathbb{H}^{n}\right) n \geq 2$ be the space of orientation preserving isometries of $\mathbb{H}^{n}$. As usual, we give the space of isometries the topology of uniform convergence on compact sets. We define a Kleinian group $\Gamma$ to be a discrete torsion-free subgroup of Isom+ $\left(\mathbb{H}^{n}\right)$. As such, $\Gamma$ acts properly discontinuously on $\mathbb{H}^{n}$, and the quotient manifold $N=\mathbb{H}^{n} / \Gamma$ is a complete Riemannian manifold of constant curvature -1 .

A Kleinian group $\Gamma$ also acts as a discrete subgroup of conformal automorphisms of the sphere at infinity $\mathbb{S}_{\infty}^{n-1}$; this action partitions $\mathbb{S}_{\infty}^{n-1}$ into two disjoint sets. The regular set $\Omega_{\Gamma}$ is the largest open set in $\mathbb{S}_{\infty}^{n-1}$ on which $\Gamma$ acts properly discontinuously, and the limit set $\Lambda_{\Gamma}$ is its complement. In the case that $\Lambda_{\Gamma}$ contains more than 2 points, it is characterized as being the smallest closed $\Gamma$-invariant subset of $\mathbb{S}_{\infty}^{n-1}$.

Define the convex hull $\mathrm{CH}\left(\Lambda_{\Gamma}\right)$ of the limit set $\Lambda_{\Gamma}$ to be the smallest convex subset of $\mathbb{H}^{n}$ so that all geodesics with both limit points in $\Lambda_{\Gamma}$ are contained in $\mathrm{CH}\left(\Lambda_{\Gamma}\right)$. We can take the quotient of $\mathrm{CH}\left(\Lambda_{\Gamma}\right)$ by $\Gamma$ (denoted by $C(\Gamma)$ ); this is the convex core. It is the smallest convex submanifold of $N=\mathbb{H}^{n} / \Gamma$ so that the inclusion map is a homotopy equivalence.

A Kleinian group is convex cocompact if its associated convex core is compact and it is geometrically finite if the volume of the unit neighborhood of the convex core is finite (see Thurston [18]). This paper deals specifically with convex cocompact 
Kleinian groups. For the basics in the theory of Kleinian groups we refer the reader to Maskit [10].

If $\Gamma$ is a geometrically finite Kleinian group, we define the space $\mathrm{QC}(\Gamma)$ of quasiconformal deformations of $\Gamma$ as follows; We consider pairs $\left(f_{0}, \Gamma_{0}\right)$ such that $f_{0}: \mathbb{S}_{\infty}^{n-1} \rightarrow \mathbb{S}_{\infty}^{n-1}$ is a quasiconformal homeomorphism, conjugating $\Gamma$ to Kleinian group $\Gamma_{0}$, ie $\Gamma_{0}=f \Gamma f^{-1}$. The map $f_{0}$ is called the marking. We define an equivalence relation by saying $\left(f_{1}, \Gamma_{1}\right) \equiv\left(f_{2}, \Gamma_{2}\right)$ if there exists a conformal map $\alpha$ conjugating $\Gamma_{1}$ to $\Gamma_{2}$, ie

$$
f_{2} \circ \gamma \circ f_{2}^{-1}=\left(\alpha \circ f_{1}\right) \circ \gamma \circ\left(\alpha \circ f_{1}\right)^{-1} \text { for all } \gamma \in \Gamma \text {. }
$$

Then $\mathrm{QC}(\Gamma)$ is the set of equivalence classes under this equivalence relation. For convenience, we will often suppress the map $f_{0}$ in describing a point of $\mathrm{QC}(\Gamma)$ and just refer to it by the group.

\subsection{Geodesic currents}

We can identify a geodesic with its endpoints on $\mathbb{S}_{\infty}^{n-1}$ and therefore we identify the space of geodesics on $\mathbb{H}^{n}$ by $G\left(\mathbb{H}^{n}\right) \cong\left(\mathbb{S}_{\infty}^{n-1} \times \mathbb{S}_{\infty}^{n-1}-\right.$ diagonal $) / \mathbb{Z}_{2}$.

If $N$ is a convex cocompact hyperbolic $n$-manifold, with $N=\mathbb{H}^{n} / \Gamma$, then each nontrivial free homotopy class of closed curve corresponds to a unique multiple of a primitive closed geodesic in $N$. If $\alpha$ is a primitive closed geodesic in $N$, we lift $\alpha$ to get a discrete subset of $G\left(\mathbb{H}^{n}\right)$ which is $\Gamma$ invariant. In this way we identify every nontrivial homotopy class of closed curves on $\mathbb{H}^{n} / \Gamma$ with a $\Gamma$ invariant discrete subset of $G\left(\mathbb{H}^{n}\right)$ and a certain integral multiplicity. We then obtain a $\Gamma$ invariant measure on $G\left(\mathbb{H}^{n}\right)$ by taking the Dirac measure on this discrete set times the multiplicity. This measure is the geodesic current associated with the closed curve. We have the following generalization:

Definition A geodesic current for Kleinian group $\Gamma$ is a positive measure on $G\left(\mathbb{H}^{n}\right)$ that is invariant under the action of $\Gamma$ and supported on the set of geodesics with endpoints belonging to limit set $\Lambda_{\Gamma}$.

As geodesic currents are Borel $\sigma$-finite measures, we can add two geodesic currents and also multiply a geodesic current by a positive constant. A geodesic current which is a constant multiple of a closed geodesic is called a discrete geodesic current.

If $\Gamma$ is a Kleinian group, we let $\mathcal{C}(\Gamma)$ be the space of geodesic currents defined for $\Gamma$. The natural topology on $\mathcal{C}(\Gamma)$, via the Radon-Riesz Representation Theorem, is the weak*-topology on the space of continuous functions with compact support in $G\left(\mathbb{H}^{n}\right)$. 
Below is a basic fact we will need concerning the topology on $\mathcal{C}(\Gamma)$. The proof involves first showing that the geodesic flow on the unit tangent bundle has the specification property [3; 15], and then applying Theorem 1 in [15].

Theorem 2.1 Let $\Gamma$ be a convex cocompact Kleinian group. Then the set of discrete geodesic currents is dense in $\mathcal{C}(\Gamma)$.

We note that if $\left[f_{0}, \Gamma_{0}\right] \in \mathrm{QC}(\Gamma)$ then $f_{0}: \Lambda_{\Gamma} \rightarrow \Lambda_{\Gamma_{0}}$ is a homeomorphism. Therefore by pushing forward measures, we obtain a continuous homeomorphism, $f_{0}: \mathcal{C}(\Gamma) \rightarrow$ $\mathcal{C}\left(\Gamma_{0}\right)$ (see Bridgeman and Taylor [5]). This map is the marking on the geodesic currents.

\subsection{Patterson-Sullivan geodesic current}

Fix $s \in \mathbb{R}^{+}$. We define the Poincaré series of a Kleinian group $\Gamma$ by

$$
g_{s}(x, y)=\sum_{\gamma \in \Gamma} e^{-s d(x, \gamma y)}
$$

where $x, y \in \mathbb{H}^{n}$ and $d$ is the hyperbolic metric on $\mathbb{H}^{n}$. Let

$$
\delta(\Gamma)=\inf \left\{s: g_{s}<\infty\right\} ;
$$

then $\delta(\Gamma)$ is called the exponent of convergence of the Poincare series. We refer the reader to Nicholls [12] for further details on the exponent of convergence.

Following the work of Patterson and Sullivan, a measure can be constructed on $\mathbb{S}_{\infty}^{n-1}$ which is supported on $\Lambda_{\Gamma}$. For $x, y \in \mathbb{H}^{n}$ and $s>\delta(\Gamma)$, we define a measure $\sigma_{x, s}$ supported on the orbit of $y$ by

$$
\sigma_{x, s}=\frac{1}{g_{s}(y, y)} \sum_{\gamma \in \Gamma} e^{-s d(x, \gamma y)} D(\gamma y)
$$

where $D(p)$ is Dirac measure at $p$. The Patterson-Sullivan measure $\sigma_{x}$ is constructed by taking a limit of these measures as $s \rightarrow \delta(\Gamma)^{+}$. The measure $\sigma_{x}$ can be used to define a measure $\tilde{m}$ on $\left(\mathbb{S}_{\infty}^{n-1} \times \mathbb{S}_{\infty}^{n-1}-\right.$ diagonal $)$ given by

$$
d \tilde{m}=\frac{d \sigma_{x}(a) d \sigma_{x}(b)}{|b-a|^{2 \delta(\Gamma)}} .
$$

We then obtain a geodesic current $m$ by taking the pushforward of $\tilde{m}$ under the $\mathbb{Z}_{2}$ cover $\pi:\left(\mathbb{S}_{\infty}^{n-1} \times \mathbb{S}_{\infty}^{n-1}-\right.$ diagonal $) \rightarrow G\left(\mathbb{H}^{n}\right)$ given by $\pi(a, b)=g$ where $g$ is the geodesics with endpoints $a, b$. This measure $m=\pi_{*}(\tilde{m})$ is $\Gamma$-invariant and supported on $\left(\Lambda_{\Gamma} \times \Lambda_{\Gamma}-\right.$ diagonal $) / \mathbb{Z}_{2}$. Therefore it is a geodesic current and is called a 
Patterson-Sullivan geodesic current for $\Gamma$. By work of Sullivan [17], for $\Gamma$ being geometrically finite, $m$ is independent of choice of $x$ up to scalar multiple.

\subsection{Length functions}

If $\Gamma$ is convex cocompact Kleinian group then associated to each element $\gamma \in \Gamma$ is a natural length function $L_{\gamma}: \mathrm{QC}(\Gamma) \rightarrow \mathbb{R}$ given by letting $L_{\gamma}\left(\left[f_{0}, \Gamma_{0}\right]\right)$ be the translation length of the element $f_{0} \circ \gamma \circ f_{0}^{-1} \in \Gamma_{0}$. This function is naturally a smooth function on $\mathrm{QC}(\Gamma)$. Similarly, if $\mu \in \mathcal{C}(\Gamma)$ is a discrete geodesic current then $\mu$ is a multiple $r$ of a closed geodesic $\alpha$. We then choose $\gamma \in \Gamma$ to be a lift of the action $\alpha$ and define $L_{\mu}$ by letting $L_{\mu}=r L_{\gamma}$.

This can be generalized for geodesic currents to obtain the following result.

Length Function Theorem (Bridgeman-Taylor [6]) Let $\Gamma$ be a convex cocompact Kleinian group acting on $\mathbb{H}^{3}$. Then there is a continuous function

$$
L: \mathcal{C}(\Gamma) \rightarrow C^{\infty}(\mathrm{QC}(\Gamma), \mathbb{R})
$$

such that $L(\mu)=L_{\mu}$ for $\mu$ a discrete geodesic current where $C^{\infty}(\mathrm{QC}(\Gamma), \mathbb{R})$ is the space of smooth real-valued functions on $\mathrm{QC}(\Gamma)$ with the $C^{\infty}$-topology.

Given $\mu \in \mathcal{C}(\Gamma)$, we define $L_{\mu}: \mathrm{QC}(\Gamma) \rightarrow \mathbb{R}$ by $L_{\mu}(X)=L(\mu, X)$. The function $L_{\mu}$ is the length function for $\mu$.

We note that the continuity of $L$ implies that if $\mu_{i} \rightarrow \mu$ then $L_{\mu_{i}} \rightarrow L_{\mu}$ uniformly on compacts subsets of $\mathrm{QC}(\Gamma)$.

\subsection{Quasifuchsian space}

Recall that a fuchsian group $\Gamma$ is a finitely generated Kleinian group which acts invariantly on an open geometric disk in $\mathbb{S}_{\infty}^{2}$. Identifying $\mathbb{S}_{\infty}^{2}$ with the extended complex plane $\widehat{\mathbb{C}}$, we consider $\Gamma$ as a group of Möbius transformations on $\widehat{\mathbb{C}}$ with limit set a subset of the extended real line $\overline{\mathbb{R}}$ such that $\Gamma$ preserves each component of $\widehat{\mathbb{C}}-\overline{\mathbb{R}}$. Then the hyperbolic plane $\mathbb{H}^{2}$ with boundary $\overline{\mathbb{R}}$ is invariant under $\Gamma$ and $S=\mathbb{H}^{2} / \Gamma$ is a hyperbolic surface.

Let $\Gamma$ be convex cocompact and fuchsian, then the quotient manifold $\mathbb{H}^{3} / \Gamma$ is homeomorphic to $S \times \mathbb{R}$, where $S$ is the closed hyperbolic surface given by $\mathbb{H}^{2} / \Gamma$. To emphasize that we are dealing with a special case, $\mathrm{QC}(\Gamma)$ is called the quasifuchsian space of $S$ and denoted by $\mathrm{QF}(S)$. Also we denote the space of currents $\mathcal{C}(\Gamma)$ by $\mathcal{C}(S)$. Furthermore we will denote the fuchsian elements of $\mathrm{QF}(S)$ by $F(S)$. 
By Bers simultaneous uniformization we have $\mathrm{QF}(S) \simeq T(S) \times T(\bar{S})$ where $T(S)$ is the Teichmüller space of $S$ and $F(S)$ corresponds to the diagonal in $T(S) \times T(\bar{S})$ (see Bers [1]). Thus if $\Delta: T(S) \rightarrow T(S) \times T(\bar{S})$ is the map $\Delta(X)=(X, \bar{X})$, then $F(S) \simeq T(S)$.

In the quasifuchsian case we have the following extension of the real length function $L$ to a complex length function $\mathcal{L}$.

Complex Length Theorem (Bridgeman-Taylor [6]) For each $\mu \in \mathcal{C}(S)$ there exists a unique holomorphic function $\mathcal{L}_{\mu}: \mathrm{QF}(S) \rightarrow \mathbb{C}$ with real part $L_{\mu}$ and imaginary part satisfying $\operatorname{Im}\left(\mathcal{L}_{\mu}\right)=0$ on $F(S)$. Furthermore the function

$$
\mathcal{L}: \mathcal{C}(S) \rightarrow C^{\omega}(\mathrm{QF}(S), \mathbb{C})
$$

given by $\mathcal{L}(\mu)=\mathcal{L}_{\mu}$ is continuous with respect to the topology of uniform convergence (on compacta) on the space $C^{\omega}(\mathrm{QF}(S), \mathbb{C})$ of holomorphic functions on $\mathrm{QF}(S)$.

Convention If $f: X \rightarrow Y$ is a smooth function then we will let $f^{\prime}(x)$ denote the derivative map $f^{\prime}(x): T_{x}(X) \rightarrow T_{f(x)}(Y)$. To simplify, if $v \in T_{x}(X)$ we will often write $f^{\prime}(v)=\left(f^{\prime}(x)\right)(v)$. Similarly if $f^{\prime}(x)=0$ then the Hessian of $f$ is denoted by $f^{\prime \prime}(x)$ and is the well-defined symmetric bilinear two-form given by

$$
\left(f^{\prime \prime}(x)\right)(v, w)=\frac{\partial^{2} f}{\partial v \partial w} .
$$

Once again we will often shorten and write $f^{\prime \prime}(v, w)=\left(f^{\prime \prime}(x)\right)(v, w)$.

\section{Weil-Petersson extension $G$}

We now describe the symmetric bilinear form $G$ on $\mathrm{QF}(S)$ given in [6].

Let $X=\left[f_{0}, \Gamma_{0}\right] \in \mathrm{QF}(S)$, then $f_{0}$ gives a natural homeomorphism $f_{0}: \mathcal{C}(S)=$ $\mathcal{C}(\Gamma) \rightarrow \mathcal{C}\left(\Gamma_{0}\right)$ between geodesic current spaces coming from the marking. We let $m_{\Gamma_{0}} \in \mathcal{C}\left(\Gamma_{0}\right)$ be a Patterson-Sullivan geodesic current and pullback to define $m_{X}=$ $f_{0}^{-1}\left(m_{\Gamma_{0}}\right) \in \mathcal{C}(S)$. We normalize to define the unit length Patterson-Sullivan geodesic current of $X$ by

$$
\mu_{X}=\frac{m_{X}}{L\left(X, m_{X}\right)}
$$

Then this geodesic current has unit length in $X$.

In [6], we show that the function $\left(h L_{\mu_{X}}\right): \mathrm{QF}(S) \rightarrow \mathbb{R}$ given by $\left(h L_{\mu_{X}}\right)(Y)=$ $h(Y) L_{\mu_{X}}(Y)$ is minimum at $X$. Using this we defined $G$ to be the symmetric bilinear form at $X$ given by

$$
G_{X}=\left(h L_{\mu_{X}}\right)^{\prime \prime}(X)
$$


Finally we proved Theorem 1.1, showing that $G$ is a natural extension of the normalized Weil-Petersson metric on $F(S)$.

\section{Thermodynamics and pressure metric}

We will now describe the pressure metric for a shift of finite type. This will be a cursory introduction to the elements of Thermodynamic Formalism needed to state and prove our results. For a complete description see the book [13] by Parry and Pollicott and the paper [11] of McMullen.

Let $A$ be a $k \times k$ matrix of zeros and ones then we define the associated (one-sided) shift of finite type by $(\Sigma, \sigma)$ where $\Sigma$ is the set of sequences

$$
\Sigma=\left\{x=\left(x_{n}\right)_{n=0}^{\infty}: x_{n} \in\{1, \ldots, k\}, A\left(x_{n}, x_{n+1}\right)=1\right\}
$$

and $\sigma: \Sigma \rightarrow \Sigma$ is the standard shift where $\sigma\left(x_{0}, x_{1}, x_{2}, \ldots\right)=\left(x_{1}, x_{2}, \ldots\right)$. We give $\{i, \ldots, k\}$ the discrete topology and $\Sigma$ the associated product topology.

The space $C(\Sigma)$ is the space of continuous real valued functions on $\Sigma$. Two functions $f, g \in C(\Sigma)$ are cohomologous $(f \sim g)$, if there exists a continuous function $h \in C(\Sigma)$ such that $f(x)-g(x)=h(\sigma(x))-h(x)$. If $f \sim 0$ then $f$ is a coboundary.

We can metrize the topology on $\Sigma$ by choosing any $K>1$ and then defining $d(x, y)=$ $K^{-N}$ where $N=N(x, y)=\min \left\{n \mid x_{n} \neq y_{n}\right\}$.

Then given $\theta \in(0,1)$ we say $f \in F_{\theta}(\Sigma)$ if there exists a constant $C>0$ such that

$$
|f(x)-f(y)| \leq C \theta^{N(x, y)} .
$$

The set $F_{\theta}$ is the set of Hölder continuous functions with the same Hölder constant, with respect to the metric $d$.

$F_{\theta}(\Sigma)$ is given the norm $\|\cdot\|_{\theta}$ by

$$
\|f\|_{\theta}=\|f(x)\|_{\infty}+\sup _{x \neq y} \frac{|f(x)-f(y)|}{\theta^{N(x, y)}} .
$$

Given a map $f$ we can take the iterated sum $S_{n} f$ defined by

$$
\left(S_{n} f\right)(x)=\sum_{k=0}^{n-1} f\left(\sigma^{k}(x)\right) .
$$

If $f \sim g$ with $f(x)-g(x)=h(\sigma(x))-h(x)$ then

$$
S_{n} f(x)-S_{n} g(x)=h\left(\sigma^{n}(x)\right)-h(x)
$$


so shifts of cohomologous functions are cohomologous. Also if $f \in F_{\theta}(\Sigma)$, the Ruelle operator $L_{f}: F_{\theta}(\Sigma) \rightarrow F_{\theta}(\Sigma)$ is defined by

$$
\left(L_{f} g\right)(x)=\sum_{\sigma(y)=x} e^{f(y)} g(y) .
$$

We note that under iteration of the Ruelle operator we have

$$
\left(L_{f}^{n} g\right)(x)=\sum_{\sigma^{n}(y)=x} e^{S_{n} f(y)} g(y) .
$$

The shift $(\Sigma, \sigma)$ is aperiodic if there exists an $n>0$ such that $A^{n}$ is all positive entries. We have the following generalization of the Perron-Frobenius Theorem for matrices.

Theorem 4.1 (Ruelle-Perron-Frobenius [13]) Let $f \in F_{\theta}(\Sigma)$ and let $(\Sigma, \sigma)$ be an aperiodic shift of finite type. Then:

(1) There is a simple maximal positive eigenvalue $\beta$ for $L_{f}$ with corresponding strictly positive eigenvector $h$.

(2) The remainder of the spectrum of $L_{f}$ is contained in a disk of radius strictly smaller than $\beta$.

(3) There is a unique probability measure $\mu$ such that $L_{f}^{*} \mu=\beta . \mu$.

(4) Let $h$ be a maximal eigenvector normalized so that $\mu(h)=1$. Then

$$
\frac{L_{f}^{n}(g)}{\beta^{n}} \longrightarrow h \int g d \mu \quad \text { uniformly for all } g \in C(\Sigma) \text {. }
$$

The pressure $P(f)$ is defined by $P(f)=\log \beta$. If $f \in F_{\theta}(\Sigma)$ satisfies $P(f)=0$ and $h$ is a maximal normalized eigenvector of $L_{f}$ then the measure $m=h . \mu$ is an ergodic $\sigma$-invariant probability measure and is called the equilibrium measure of $f$.

In [13] the properties of the function $P: F_{\theta}(\Sigma) \rightarrow F_{\theta}(\Sigma)$ are described in detail. In particular it is convex and real-analytic and depends only on cohomology class.

Also if $P(f)=0$, with equilibrium measure $m$ and $g \in F_{\theta}(\Sigma)$ then

$$
P^{\prime}(f)(g)=\left.\frac{d}{d t} P(f+t g)\right|_{t=0}=\int g d m .
$$

Also if $P^{\prime}(f)(g)=0$ then the variance $\operatorname{Var}(g, m)$ is defined by

$$
P^{\prime \prime}(f)(g)=\left.\frac{d^{2}}{d t^{2}} P(f+t g)\right|_{t=0}=\operatorname{Var}(g, m) .
$$


We define $T(\Sigma)$ to be the set of pressure zero, Hölder continuous functions up to coboundary, that is

$$
T(\Sigma)=\left\{f: f \in F_{\theta}(\Sigma) \text { for some } \theta, P(f)=0\right\} / \sim .
$$

If $[f] \in T(\Sigma)$ and $f$ has equilibrium measure $m$, then by the formula for the derivative of pressure $P$, the tangent space of $T(\Sigma)$ at $[f]$ can be identified with

$$
T_{[f]} T(\Sigma)=\left\{g: \int g d m=0\right\} / \sim .
$$

The pressure metric $\|\cdot\|_{P}$ on $T(\Sigma)$ is then defined by

$$
\|[g]\|_{P}=\frac{\operatorname{Var}(g, m)}{-\int f d m} .
$$

By Theorem 4.2 of [13], $\operatorname{Var}(g, m)=0$ implies that $g \sim 0$. Thus $\|[g]\|_{P}=0$ implies $[g]=0$ and therefore $\|\cdot\|_{P}$ is positive definite metric on $T(\Sigma)$.

\section{Thermodynamics on $\mathrm{QF}(S)$}

Let $\Gamma$ be a quasifuchsian group with limit set $\Lambda_{\Gamma} \subset \widehat{\mathbb{C}}$. A conformal Markov map for $\Gamma$ is a piecewise conformal map $f: \Lambda_{\Gamma} \rightarrow \Lambda_{\Gamma}$ such that $\Lambda_{\Gamma}$ has a partition into segments $J_{1}, \ldots, J_{m}$ so that

(1) $\left.f\right|_{J_{k}}=\left.\gamma_{k}\right|_{J_{k}}$ for some $\gamma_{k} \in \Gamma$,

(2) for each $k, f\left(J_{k}\right)$ is the union of various $J_{l}$ 's.

A Markov map is expanding if there is an $n>0$ such that the n-th iterate $f^{n}=$ $f \circ f \circ \ldots \circ f$ has derivative whose norm in the spherical metric satisfies

$$
\left|\left(f^{n}\right)^{\prime}(x)\right|>C>1
$$

and for any $U \subset L_{\Gamma}$ open, there exists an $m>0$ such that $f^{m}(U)=\Lambda_{\Gamma}$.

If $\Gamma$ has an expanding Markov map $f$ then we can define a matrix $A$ by $A(i, j)=1$ if $J_{j} \subset f\left(J_{i}\right)$ and zero otherwise. Then we have an aperiodic shift $(\Sigma, \sigma)$ and we define $\pi: \Sigma \rightarrow L_{\Gamma}$ by $\pi(x)=z$ where $f^{i}(z) \in J_{x_{i}}$. The map $f$ obviously satisfies $f(\pi(x))=\pi(\sigma(x))$. The map $\pi$ is surjective but as the segments $J_{i}$ may have boundary points in common, the map $\pi$ is two to one on a countable set of points $P$. If $Q$ is the finite set of endpoints of the $J_{i}$ 's then $P$ is precisely

$$
P=\bigcup_{n=0}^{\infty} f^{-n}(Q) \text {. }
$$


The points of $P$ are called $b a d$ points and if $z \notin P$ it is called a good point. We note that if $z$ is a good point, then there is a unique $x \in \Sigma$ such that $\pi(x)=z$ and for any $n>0$, there is unique $\gamma_{n} \in \Gamma$ such that $f^{n}=\gamma_{n}$ on an open interval about $z$.

\subsection{Expanding Markov map for quasifuchsian groups}

In the following we describe Bowen's results from [4] on expanding Markov maps for quasifuchsian groups.

Bowen first considered the cocompact fuchsian group $\Gamma_{r}$ obtained by identifying sides of a regular hyperbolic $4 n$-gon in the standard way given by the side labelling

$$
x_{1} y_{1} x_{1}^{-1} y_{1}^{-1} \cdots x_{n} y_{n} x_{n}^{-1} y_{n}^{-1} \text {. }
$$

He then described an expanding Markov map $f_{\Gamma_{r}}: \mathbb{S}^{1} \rightarrow \mathbb{S}^{1}$ for $\Gamma_{r}$ which we will describe in detail below.

Then if $g: \widehat{\mathbb{C}} \rightarrow \widehat{\mathbb{C}}$ is a quasiconformal map conjugating the action of $\Gamma_{r}$ to the action of $\Gamma$, then this gives the map $f_{\Gamma}: \Lambda_{\Gamma} \rightarrow \Lambda_{\Gamma}$ by $f_{\Gamma}=g \circ f_{\Gamma_{r}} \circ g^{-1}$ and $\pi_{\Gamma}: \Sigma \rightarrow \Lambda_{\Gamma}$ by $\pi_{\Gamma}=g \circ \pi_{\Gamma_{r}}$. Then $f_{\Gamma}$ is an expanding Markov map for $\Gamma$ with the same shift space $(\Sigma, \sigma)$.

The function $\phi_{\Gamma}: \Sigma \rightarrow \mathbb{R}$ defined by $\phi_{\Gamma}(x)=-\log \left|f_{\Gamma}^{\prime}\left(\pi_{\Gamma}(x)\right)\right|$ is Hölder continuous. By the chain rule for differentiation we have

$$
\left(S_{n} \phi_{\Gamma}\right)(x)=-\log \left|\left(f_{\Gamma}^{n}\right)^{\prime}\left(\pi_{\Gamma}(x)\right)\right| .
$$

Then if $h_{\Gamma}$ is the Hausdorff dimension of the limit set $\Lambda_{\Gamma}$, Bowen showed that $h_{\Gamma}$ is characterized by the equation

$$
P\left(h_{\Gamma} \phi_{\Gamma}\right)=0 .
$$

We now describe the map $f_{\Gamma_{r}}$ in more detail. The group $\Gamma_{r}$ has fundamental domain $D$, the regular hyperbolic $4 n$-gon. We label the sides of $D$ by $s_{i}, i=1, \ldots, 4 n$. Each $s_{i}$ belongs to a unique geodesic $g_{i}$ with endpoints $p_{i}, q_{i}$ on $\mathbb{S}^{1}$. We let $I_{i}$ be the interval on $\mathbb{S}^{1}$ with endpoints $p_{i}, q_{i}$ which is smallest in length. We further define $\gamma_{i} \in \Gamma_{r}$ to be the element which identifies $s_{i}$ with another side $s_{j}$ of $D$ for some $j$.

For each $\gamma \in \Gamma_{r}$ we let $D_{\gamma}=\gamma(D)$ and say $D_{\gamma}$ abuts $D$ if $D_{\gamma} \cap D \neq \varnothing$. For each $D_{\gamma}$ we define the intervals $I_{\gamma, i}=\gamma\left(I_{i}\right)$. We let $R \subseteq \mathbb{S}^{1}$ be the union of the endpoints of $I_{\gamma, i}$ for $D_{\gamma}$ abutting $D$. Then $R$ defines a decomposition of $\mathbb{S}^{1}$ into intervals $J_{k}$. We note that each $J_{k} \subseteq I_{j}$ for some $j$, not necessarily unique. We then define

$$
\left.f_{\Gamma_{r}}\right|_{J_{k}}=\gamma_{j} \text { where } J_{k} \subseteq I_{j} .
$$


It follows that for any choice of $j$ such that $J_{k} \subseteq I_{j}$, the map $f_{\Gamma_{r}}$ is a Markov map for $\Gamma_{r}$. Bowen makes a specific choice to define $f_{\Gamma_{r}}: \mathbb{S}^{1} \rightarrow \mathbb{S}^{1}$ such that $f_{\Gamma_{r}}$ is an expanding Markov map and orbit equivalent to the action of $\Gamma_{r}$ on $\mathbb{S}^{1}$ (see Bowen [4]). We say a geodesic $g$ abuts $D$ if it intersects one of the images of $D$ in the tessellation that abuts $D$. We now describe an elementary property of the map $f_{\Gamma_{r}}$ that follows easily from its definition.

Lemma 5.1 Let $f_{\Gamma_{r}}: \mathbb{S}^{1} \rightarrow \mathbb{S}^{1}$ be the expanding map for the $\Gamma_{r}$ described above. Let $g$ be a geodesic with endpoints $a, b$ that abuts $D$. If $f_{\Gamma_{r}}=\gamma \in \Gamma_{r}$ at $a$, then geodesic $\gamma(g)$ abuts $D$.

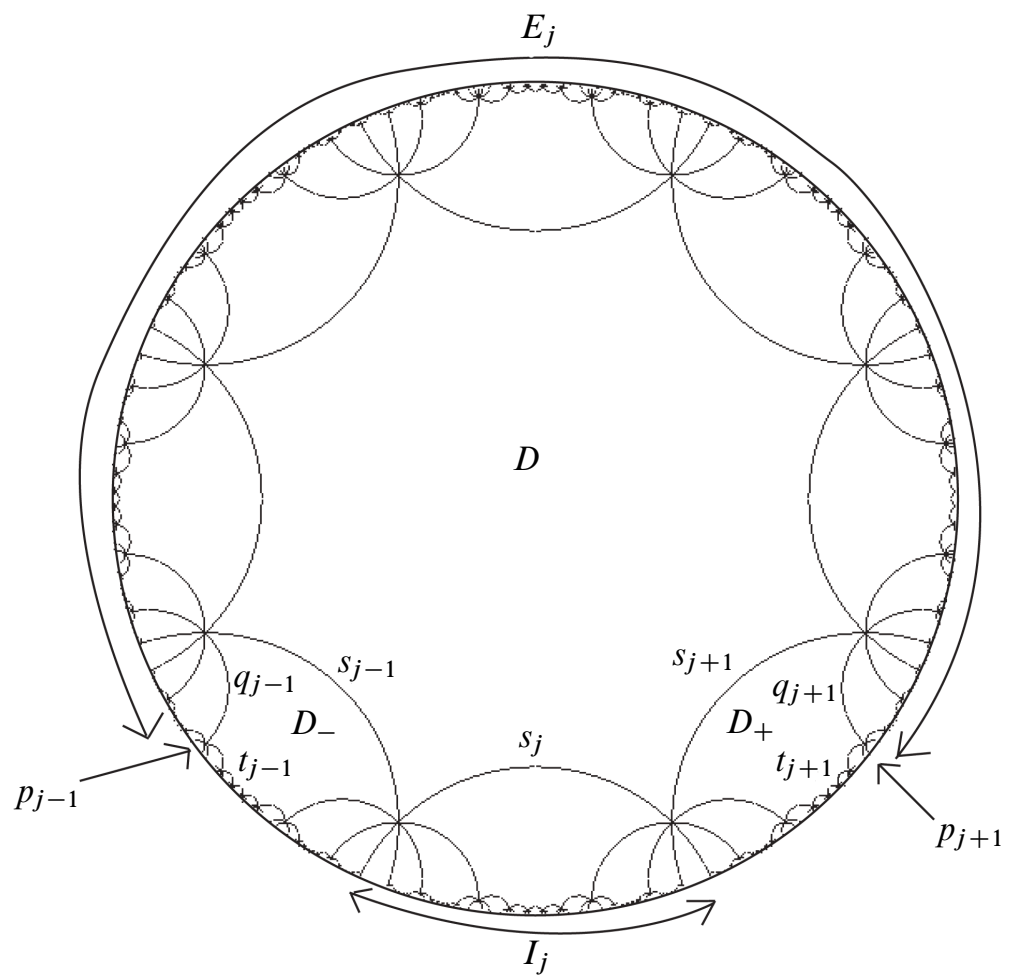

Figure 1: Tesselation by regular $4 n-$ gons

Proof We place $D$ with the origin in the center. We label the edges $e_{i}$ of $D$ clockwise for $i=1, \ldots, 4 n$. The edges $e_{i}$ define geodesics $g_{i}$ which given overlapping intervals $I_{i}$. We further define the half-plane given by $I_{i}, H_{i}$ (see Figure 1).

Let $g$ be a geodesic with endpoints $a, b$ which abuts $D$ and $f_{\Gamma_{r}}=\gamma$ at $a$. Then $\gamma=\gamma_{j}$ for some $j$ and $a \in I_{j}$. We let $P$ be the convex polygon obtained by taking the union of all domains that abut $D$. Then by assumption $g$ intersects $P$. 
If $s_{j}$ is the side of $D$ corresponding to the side identification $\gamma_{j}$, we let $P_{1}$ be the collection of domains which intersect $s_{j}$ (ie contain $s_{j}$ as a side or contain an endpoint of $s_{j}$ as a vertex). As $\gamma_{j}\left(s_{j}\right)=s_{k}$ some other side of $D$, if $g$ intersects $P_{1}$ then $\gamma_{j}(g)$ abuts $D$. We will now show that if $g$ intersects $P$ then it intersects $P_{1}$ thereby proving the result.

Let $P_{2}$ be the set of domains $P \cap H_{j}$. As $H_{j} \cap D=s_{j}$, then $P_{2} \cap P_{1}$. As we are assuming $g$ does not intersect $P_{1}$, then $g$ does not intersect $P_{2}$. If both endpoints of $g$ are in $I_{j}$ then by convexity $g \subseteq H_{j}$ and therefore $\varnothing \neq g \cap P=g \cap\left(P \cap H_{j}\right)=$ $g \cap P_{2} \subseteq g \cap P_{1}$. Thus if $g$ has both endpoints in $I_{j}$ then $g$ intersects $P_{1}$.

We let $D_{-}$be the domain that shares the side $s_{j-1}$ with $D$ and $D_{+}$be the domain that shares the side $s_{j+1}$ with $D$. Then as $s_{j-1}, s_{j+1}$ share a vertex with $s_{j}$ then $D_{-}, D_{+} \subseteq P_{1}$.

In domain $D_{-}$we label the opposite side to $s_{j-1}$ by $t_{j-1}$. We let $h_{j-1}$ be the geodesic associated to $t_{j-1}$ and the interval $T_{j-1} \subseteq I_{j-1}$. The interval $T_{j-1}$ does not intersect with any other interval $I_{k}$ for $k \neq j-1$. Also $D_{-}$shares a unique side with a domain of $P-P_{1}$ (abutting $D$ but not side $s_{j}$ ). We label the geodesic to the side $q_{j-1}$ and its unique endpoint $p_{j-1} \in I_{j-1}$. We define similar quantities for $D_{+}$. We let $E_{j}$ be the interval in $\mathbb{S}^{1}$ with endpoints $p_{j-1}, p_{j+1}$ and not containing $I_{j}$. Then $E_{j}$ is disjoint from $T_{j-1}$ and $T_{j+1}$. If $g$ intersects a domain of $P$ but not of $P_{1}$ then $g$ must have an endpoint in $E_{j}$. Therefore $g$ separates the geodesics $h_{j-1}, h_{j+1}$. Therefore $g$ must intersect either $D_{-}, D_{+}$or $D$. Thus $g$ intersects $P_{1}$.

The above Lemma says that the abutting geodesics are an invariant set under the Möbius map defined by their endpoints.

We now prove an important property of the expanding Markov map $f_{\Gamma_{r}}$ that we will need later.

If $G$ is a group, then we say $g$ is commensurable to $h$ if there exists $k \in G$ such that $g^{n}=k h^{m} k^{-1}$ for some $n, m \neq 0$. The set of commensurability classes of $G$ is denoted $[G]$. We note that for $\Gamma$ a cocompact Kleinian group, then $[\Gamma]$ is equivalent to the set of primitive geodesics in $N=\mathbb{H}^{n} / \Gamma$.

Lemma 5.2 Let $f_{\Gamma_{r}}: \mathbb{S}^{1} \rightarrow \mathbb{S}^{1}$ be the expanding Markov map described above. Then there is a finite set $\mathcal{S} \subseteq\left[\Gamma_{r}\right]$ of commensurability classes such that if $[\gamma] \notin \mathcal{S}$, then:

(1) If $\gamma^{\prime} \in[\gamma]$ then the endpoints of the axis of $\gamma^{\prime}$ are good points of $f_{\Gamma_{r}}$.

(2) There exists a $\gamma^{\prime} \in[\gamma]$ whose axis abuts $D$ and has fixed points $a, b$ such that the expanding fixed point $a$ of $\gamma^{\prime}$ is a periodic point of $f_{\Gamma_{r}}$. 
Proof We note that as $\Gamma_{r}$ does not contain parabolics, then if two elements $\gamma_{1}, \gamma_{2}$ have a common fixed point, then they share the same axis and are commensurate. Let $D$ be the fundamental domain for $\Gamma_{r}$ given by the regular $4 n$-gon in the Poincare disk model with center at 0 . We extend the $4 n$ sides of $D$ to complete geodesics $g_{i}$, $i=1, \ldots, 4 n$, and let $P_{0}$ be the union of the endpoints of the $g_{i}$ 's. We then define $P=\Gamma_{r} P_{0}$, the orbit of $P_{0}$ under the group. The set $P$ is precisely the set of bad points for $f_{\Gamma_{r}}$. Also as $D$ is the regular $4 n$-gon, each of the geodesics $g_{i}$ is the axis for an element of $\Gamma$ and we let $\gamma_{i}$ be the corresponding primitive element. Then we let $\mathcal{S}=\left\{\left[\gamma_{i}\right]\right\}_{i=1}^{4 n}$. If an element $\gamma \in \Gamma_{r}$ has a bad endpoint $z \in P$, then $z=\gamma_{1} . z_{0}$ for $z_{0} \in P_{0}$ then $\gamma_{1}^{-1} \gamma \gamma_{1}$ has fixed point $z_{0}$. Therefore $\gamma_{1}^{-1} \gamma \gamma_{1}$ shares an endpoint with some $\gamma_{i}$. Therefore $\gamma$ and $\gamma_{i}$ are commensurate. Thus we conclude that if $[\gamma] \notin \mathcal{S}$, then $\gamma$ has good endpoints.

We now let $[\gamma] \notin \mathcal{S}$ and choose $\gamma$ such that its axis intersects $D$. As $[\gamma] \notin \mathcal{S}$, then its axis $g$ has endpoints $a_{0}, b_{0}$. We define geodesic $g_{n}$ to have endpoints $a_{n}=f_{\Gamma_{r}}^{n}(a)$, and let $b_{n} \in \mathbb{S}^{1}$ be the unique point such that the pair $\left(a_{n}, b_{n}\right) \in \mathbb{S}^{1} \times \mathbb{S}^{1}$ are endpoints of the axis of a conjugate of $\gamma$. Then by Lemma 5.1, $g_{n}$ also abuts $D$. By compactness of the union of domains abutting $D$, we have there is an $\epsilon>0$ such that $\left|a_{n}-b_{n}\right|>\epsilon$ for all $n$. But if the sequence $\left\{\left(a_{n}, b_{n}\right)\right\}$ in $\mathbb{S}^{1} \times \mathbb{S}^{1}$ has an infinite number of values, it must have a convergent subsequence. As the orbit of the axis $g$ under $\Gamma_{r}$ is discrete in the space of geodesics $G\left(\mathbb{H}^{2}\right)$, any convergent subsequence must converge to a point on the diagonal of $\mathbb{S}^{1} \times \mathbb{S}^{1}$ contradicting $\left|a_{n}-b_{n}\right|>\epsilon$. Therefore the sequence takes a finite set of values and there exists a $k>0$ and an $n$ such that $\left(a_{n}, b_{n}\right)=\left(a_{n+k}, b_{n+k}\right)$ and therefore $f_{\Gamma_{r}}^{k}\left(a_{n}\right)=a_{n}$ and $a_{n}$ is a periodic point for $f_{\Gamma_{r}}$. We let $\gamma^{\prime}$ be the conjugate of $\gamma$ with endpoints $\left(a_{n}, b_{n}\right)$, giving the result.

\subsection{Pullback of pressure metric}

We first define the Hausdorff dimension function $h: \mathrm{QF}(S) \rightarrow \mathbb{R}$ by $h\left(\left[f_{0}, \Gamma_{0}\right]\right)=h_{\Gamma_{0}}$, the Hausdorff dimension of the limit set $\Lambda_{\Gamma_{0}}$. This is well-defined, and by Ruelle [14], $h$ is real-analytic.

We let $\Gamma$ be a fuchsian group such that $S=\mathbb{H}^{2} / \Gamma$ with expanding Markov map $f_{\Gamma}$ as described in the Section 5.1. We let $(\Sigma, \sigma)$ be the associated shift and $\pi_{\Gamma} ; \Sigma \rightarrow \mathbb{S}^{1}$ as before. Then for each $X \in \mathrm{QF}(S)$ we let $X=\left[g_{0}, \Gamma_{0}\right]$ where $g_{0}$ conjugates $\Gamma$ to $\Gamma_{0}$. Then we define $\phi_{X}=\phi_{\Gamma_{0}}: \Sigma \rightarrow \mathbb{R}$ and $\Phi_{X}=h_{\Gamma_{0}} \phi_{\Gamma_{0}}$.

Note that if $\left[g_{0}, \Gamma_{0}\right]=\left[g_{1}, \Gamma_{1}\right]$, then $\phi_{\Gamma_{0}}, \phi_{\Gamma_{1}}$ are cohomologous (see McMullen [11]). Also, by Equation (4), $P\left(\Phi_{X}\right)=0$. Thus we obtain a well-defined map $F: \mathrm{QF}(S) \rightarrow$ $T(\Sigma)$ by $F(X)=\left[\Phi_{X}\right]$. We then define $W$ to be the pullback of the pressure metric 
on $T(\Sigma)$. As the pressure metric is positive definite it follows that $W$ is at least nonnegative.

To obtain a formula for $\|\cdot\|_{W}$, given $v \in T_{X}(\mathrm{QF}(S))$, we choose a smooth curve $\alpha:(-\epsilon, \epsilon) \rightarrow \mathrm{QF}(S)$ with $\alpha(0)=X$ and $\alpha^{\prime}(0)=v$. Then let $\alpha(t)=X_{t}=\left[g_{t}, \Gamma_{t}\right]$ where $g_{t}$ is a smooth 1 -parameter family of quasiconformal maps conjugating $\Gamma$ to $\Gamma_{t}$.

We let $\Phi_{t}=\Phi_{\alpha(t)}$ and $\phi_{t}=\phi_{\alpha(t)}$ and define $\dot{\Phi}_{0}$ by

$$
\dot{\Phi}_{0}(x)=\left.\frac{d}{d t}\right|_{t=0}\left(\Phi_{t}(x)\right)
$$

Then by definition of the pressure metric in Equation (2), $\|v\|_{W}$ is given by

$$
\|v\|_{W}^{2}=\frac{\operatorname{Var}\left(\dot{\Phi}_{0}, m\right)}{-\int \Phi_{0} d m}
$$

We obtain an alternative definition of $\|\cdot\|_{W}$ by noting that $P\left(\Phi_{t}\right)=0$ and taking derivatives with respect to $t$. Taking first derivatives we obtain

$$
P^{\prime}\left(\Phi_{t}\right)\left(\dot{\Phi}_{t}\right)=0
$$

Then taking derivative again we have

$$
P^{\prime \prime}\left(\Phi_{t}\right)\left(\dot{\Phi}_{t}\right)+P^{\prime}\left(\Phi_{t}\right)\left(\ddot{\Phi}_{t}\right)=0 .
$$

Evaluating at $t=0$ we have

$$
\operatorname{Var}\left(\dot{\Phi}_{0}, m\right)+\int \ddot{\Phi}_{0} d m=0
$$

Therefore we have

$$
\|v\|_{W}^{2}=\frac{\operatorname{Var}\left(\dot{\Phi}_{0}, m\right)}{-\int \Phi_{0} d m}=\frac{\int \ddot{\Phi}_{0} d m}{\int \Phi_{0} d m} .
$$

\section{Conformal equivalence of $G$ and $W$}

The proof that $G$ and $W$ are conformally equivalent follows by generalizing the argument in [11] of McMullen for the fuchsian subspace $F(S)$ to all of quasifuchsian space $\mathrm{QF}(S)$.

Theorem 6.1 The pseudometrics $G$ and $W$ are conformally equivalent with

$$
\|v\|_{G}=\sqrt{h(X)} \cdot\|v\|_{W} \text { for } v \in T_{X}(\mathrm{QF}(S)) .
$$


Proof From Equation (5), we have

where

$$
\begin{gathered}
\|v\|_{W}^{2}=\frac{\int \ddot{\Phi}_{0} d m}{\int \Phi_{0} d m}=\frac{\left.\frac{d^{2}}{d t^{2}}\left(h\left(X_{t}\right) \int \phi_{t} d m\right)\right|_{t=0}}{h(X) \int \phi_{0} d m}=\frac{1}{h(X)}(h F)^{\prime \prime}(v) \\
F(t)=\frac{\int \phi_{t} d m}{\int \phi_{0} d m} .
\end{gathered}
$$

Therefore the result follows from showing that $F(t)=L_{\mu_{X}}(X(t))$ where $\mu_{X} \in \mathcal{C}(S)$ is the unit Patterson-Sullivan geodesic current for $X$.

By the density of discrete geodesic currents (Theorem 2.1), there exists a sequence of discrete geodesic currents $\mu_{n}$ such that $\mu_{n} \rightarrow \mu_{X}$. As $\mu_{X}$ is unit length in $X$ we can normalize so that $\mu_{n}$ are unit length in $X$. Therefore $\mu_{n}=\alpha_{n} / l_{n}$ where $\alpha_{n}$ is a geodesic current coming from Dirac measure on the lifts of a primitive geodesic (also labeled $\alpha_{n}$ ) and $l_{n}$ is the length of $\alpha_{n}$ in $X$.

We choose as our basepoint for $\mathrm{QF}(S)$ the fuchsian group $\Gamma=\Gamma_{r}$ described in Section 5.1. By Lemma 5.2 for each $\alpha_{n}$ we can choose a lift $\gamma_{n} \in \Gamma$ such that the axis $g_{n}$ of $\gamma_{n}$ abuts $D$ and has fixed points $a_{n}, b_{n}$ with expanding fixed point $a_{n}$ being a periodic point for $f_{\Gamma}$ with period $p_{n}$.

As $f_{\Gamma}^{p_{n}}\left(a_{n}\right)=a_{n}$, then $f_{\Gamma}^{p_{n}}=\gamma \in \Gamma$ in an open neighborhood of $a_{n}$ where $\gamma$ fixes $a_{n}$. Then $\gamma$ and $\gamma_{n}$ both fix $a_{n}$ and therefore are commensurate with axes being equal. As $\gamma_{n}$ is primitive, it follows that $\gamma=\gamma_{n}^{k_{n}}$ for some nonzero integer $k_{n}$. Letting $X(t)=\left[g_{t}, \Gamma_{t}\right]$, then $g_{t}\left(a_{n}\right)$ is a fixed point of $f_{\Gamma_{t}}^{p_{n}}$. Also if we let $\gamma_{n, t}=g_{t} \circ \gamma_{n} \circ g_{t}^{-1}$ then $\gamma_{n, t}$ has fixed point $g_{t}\left(a_{n}\right)$ and $f_{\Gamma_{t}}^{p_{n}}=\left(\gamma_{n, t}\right)^{k_{n}}$ in an open neighborhood of $g_{t}\left(a_{n}\right)$.

Then for $i=0, p_{n}-1$ we let $g_{n}(i)$ be the element of the orbit of $g_{n}$ with endpoints $a_{n}(i)$ and $b_{n}(i)$, where $a_{n}(i)=f_{\Gamma}^{i}\left(a_{n}\right)$. Since geodesic $g_{n}$ abuts $D$, then by Lemma 5.1, $g_{n}(i)$ must also abut $D$. Therefore by compactness of the finite union of domains abutting $D$, there is an $\epsilon>0$ such that $\left|a_{n}(i)-b_{n}(i)\right|>\epsilon$ for all $n, i$.

We let $m_{n}$ be the probability measure on $\mathbb{S}^{1}$ obtained by taking $1 / p_{n}$ dirac measure on the $a_{n}(i), i=0, \ldots, p_{n-1}$.

We have $\phi_{t}: \Sigma \rightarrow \mathbb{R}$ is given by $\phi_{t}(x)=-\log \left|f_{\Gamma_{t}}^{\prime}\left(\pi_{t}(x)\right)\right|$ where $f_{\Gamma_{t}}=g_{t} \circ f_{\Gamma} \circ g_{t}^{-1}$ and $\pi_{t}=g_{t} \circ \pi_{\Gamma}$. Therefore $\phi_{t}(x)=\bar{\phi}_{t}\left(\pi_{\Gamma}(x)\right)$ where $\bar{\phi}: \mathbb{S}^{1} \rightarrow \mathbb{R}$ is the map $\bar{\phi}_{t}(z)=-\log \left|f_{\Gamma_{t}}^{\prime}\left(g_{t}(z)\right)\right|$. Then

$$
m_{n}\left(\bar{\phi}_{t}\right)=\int_{\mathbb{S} 1} \bar{\phi}_{t}(z) d m_{n}=-\frac{1}{p_{n}} \log \left|\left(f_{\Gamma_{t}}^{p_{n}}\right)^{\prime}\left(g_{t}\left(a_{n}\right)\right)\right| .
$$


As $g_{t}\left(a_{n}\right)$ is a fixed point of $f_{\Gamma_{t}}^{p_{n}}$ with $f_{\Gamma_{t}}^{p_{n}}=\gamma_{t}^{k_{n}}$ in an open neighborhood of $g_{t}\left(a_{n}\right)$ we have

$$
m_{n}\left(\bar{\phi}_{t}\right)=\int_{\mathbb{S}^{1}} \bar{\phi}_{t} d m_{n}=-\frac{1}{p_{n}} \log \left|\left(\left(\gamma_{n, t}\right)^{k_{n}}\right)^{\prime}\left(g_{t}\left(a_{n}\right)\right)\right|=-\frac{k_{n}}{p_{n}} L_{\gamma_{n}}\left(X_{t}\right) .
$$

In particular we have

Therefore

$$
\begin{aligned}
& m_{n}\left(\bar{\phi}_{0}\right)=-\frac{k_{n}}{p_{n}} L_{\gamma_{n}}(X)=-\frac{k_{n} l_{n}}{p_{n}} . \\
& \frac{m_{n}\left(\bar{\phi}_{t}\right)}{m_{n}\left(\bar{\phi}_{0}\right)}=\frac{L_{\gamma_{n}}\left(X_{t}\right)}{L_{\gamma_{n}}(X)}=L_{\mu_{n}}\left(X_{t}\right) .
\end{aligned}
$$

We now show that $l_{n} / p_{n}$ is bounded. As the map $\bar{\phi}_{0}$ is bounded on $\mathbb{S}^{1}$, there exists a $C$ such that $\left|\bar{\phi}_{0}\right| \leq C$. As $\mu_{n}$ is a probability measure and $k_{n}$ is a nonzero integer,

$$
\frac{l_{n}}{p_{n}} \leq\left|\frac{k_{n} l_{n}}{p_{n}}\right| \leq\left|\int \bar{\phi}_{0} d m_{n}\right| \leq \int\left|\bar{\phi}_{0}\right| d m_{n} \leq C .
$$

Let $v_{n}$ be the probability measure on $G\left(\mathbb{H}^{3}\right)$ obtained by taking $1 / p_{n}$ times Dirac measure on set of geodesics $g_{n}(i)$ given by the endpoint pair $\left(a_{n}(i), b_{n}(i)\right)$. As $\left|a_{n}(i)-b_{n}(i)\right|>\epsilon$ for all $n, i$, the measures $v_{n}$ does not accumulate on the diagonal, therefore the sequence $v_{n}$ has convergent subsequences in the weak* topology on $G\left(\mathbb{H}^{3}\right)$. Let $v$ be a limit with $v=\lim _{i \rightarrow \infty} v_{n_{i}}$.

We will show that $v$ is absolutely continuous with respect to $\mu_{X}$. Let $A$ be a set with $\mu_{X}(A)=0$, then as $\mu_{n} \rightarrow \mu_{X}$

$$
\lim _{n \rightarrow \infty} \mu_{n}(A)=\mu_{X}(A)=0 .
$$

We compare $\mu_{n}$ and $v_{n}$. Both are discrete measures and the support of $v_{n}$ is contained in the support of $\mu_{n}$ and with measures $v_{n}, \mu_{n}$ having point masses $1 / p_{n}, 1 / l_{n}$ respectively. Therefore by Equation (6)

$$
v_{n}(A) \leq \frac{l_{n}}{p_{n}} \mu_{n}(A) \leq C \mu_{n}(A) .
$$

Thus we have

$$
v(A)=\lim _{i \rightarrow \infty} v_{n_{i}}(A) \leq \lim _{i \rightarrow \infty} C \mu_{n_{i}}(A) \leq C \mu_{X}(A)=0 .
$$

Thus $\mu_{X}(A)=0$ implies $v(A)=0$. Thus $v$ is absolutely continuous with respect to $\mu_{X}$. We take $m_{n_{i}}$ to be the probability measures corresponding to the convergent 
sequence $v_{n_{i}}$. By reducing to subsequence we can assume that $\mu_{n_{i}}$ converge to a probability measure $m_{f}$ on $\mathbb{S}^{1}$. Then we have that $m_{f}$ satisfies

$$
m_{f}=\lim _{i \rightarrow \infty} m_{n_{i}}
$$

and

$$
\frac{m_{f}\left(\bar{\phi}_{t}\right)}{m_{f}\left(\bar{\phi}_{0}\right)}=\lim _{i \rightarrow \infty} \frac{m_{n_{i}}\left(\bar{\phi}_{t}\right)}{m_{n_{i}}\left(\bar{\phi}_{0}\right)}=\lim _{i \rightarrow \infty} L_{\mu_{n_{i}}}\left(X_{t}\right)=L_{\mu_{X}}\left(X_{t}\right) .
$$

Let $g_{0}: \mathbb{S}^{2} \rightarrow \mathbb{S}^{2}$ be the quasiconformal homeomorphism conjugating $\Gamma$ to $\Gamma_{0}$. Then by the definition of the Patterson-Sullivan geodesic current $\mu_{X}$ (see Equation (1)) we have

$$
\left(d \mu_{X}\right)(a, b)=\pi_{*}\left(\frac{d m_{X} d m_{X}}{\left|g_{0}(a)-g_{0}(b)\right|^{2 h(X)}}\right) .
$$

where $m_{X}$ is the Patterson-Sullivan measure for $\Gamma_{0}$ and $\pi$ is the $\mathbb{Z}_{2}$ cover $\pi$ : $\left(\mathbb{S}^{2} \times\right.$ $\mathbb{S}^{2}-$ diagonal $) \rightarrow G\left(\mathbb{H}^{3}\right)$. Therefore $\mu_{X}$ is absolutely continuous with respect to the measure $\pi_{*}\left(m_{X} \times m_{X}\right)$ on $G\left(\mathbb{H}^{3}\right)$.

Now we will show that $m_{f}$ is absolutely continuous with respect to $m_{X}$. Let $m_{X}(A)=$ 0 . If $S \subset \mathbb{S}^{2} \times \mathbb{S}^{2}$, we let $[S]=\pi(S-$ diagonal $)$. Then $[S]$ is precisely the set of (unoriented) geodesics in $S$. Then by definition of $m_{n}$ we have that

$$
m_{n}(A)=v_{n}\left(\left[A \times \mathbb{S}^{2}\right]\right) .
$$

As $m_{X}(A)=0$ then $\left(m_{X} \times m_{X}\right)\left(A \times \mathbb{S}^{2}\right)=\left(m_{X} \times m_{X}\right)\left(\mathbb{S}^{2} \times A\right)=0$. Therefore on $G\left(\mathbb{H}^{3}\right)$ we obtain

$$
\begin{aligned}
\pi_{*}\left(m_{X} \times m_{X}\right)\left(\left[A \times \mathbb{S}^{2}\right]\right) & =m_{X} \times m_{X}\left(\pi^{-1}\left(\left[A \times \mathbb{S}^{2}\right]\right)\right) \\
& =m_{X} \times m_{X}\left(\left(\left(A \times \mathbb{S}^{2}\right) \cup\left(\mathbb{S}^{2} \times A\right)\right)-\text { diagonal }\right) \\
& \leq\left(m_{X} \times m_{X}\right)\left(A \times \mathbb{S}^{2}\right)+\left(m_{X} \times m_{X}\right)\left(\mathbb{S}^{2} \times A\right)=0 .
\end{aligned}
$$

Therefore $\pi_{*}\left(m_{X} \times m_{X}\right)\left(\left[A \times \mathbb{S}^{2}\right]\right)=0$ and as $\mu_{X}$ is absolutely continuous with respect to $\pi_{*}\left(m_{X} \times m_{X}\right)$, then $\mu_{X}\left(\left[A \times \mathbb{S}^{2}\right]\right)=0$. Then as $v$ is absolutely continuous with respect to $\mu_{X}$ we have $v\left(\left[A \times \mathbb{S}^{2}\right]\right)=0$. As $m_{n}(A)=v_{n}\left(\left[A \times \mathbb{S}^{2}\right]\right)$ then

$$
m_{f}(A)=\lim _{i \rightarrow \infty} m_{n_{i}}(A)=\lim _{i \rightarrow \infty} v_{n_{i}}\left(\left[A \times \mathbb{S}^{2}\right]\right)=v\left(\left[A \times \mathbb{S}^{2}\right]\right)=0 .
$$

Therefore the limit $m_{f}$ must be absolutely continuous with respect to $m_{X}$. By Sullivan [16], the Patterson-Sullivan measure $m_{X}$ is equal to the Hausdorff measure of dimension $h(X)$ on the limit set. Also by Bowen the pushforward $\bar{m}=\left(\pi_{\Gamma_{0}}\right)_{*}(m)$ of the equilibrium measure $m$ to $\mathbb{S}^{1}$ is equivalent to the Hausdorff measure of dimension $h(X)$ on the limit set and therefore equivalent to $m_{X}$ [4, Lemma 10]. Therefore $m_{f}$ is then absolutely continuous with respect to the measure $\bar{m}$. Also as the $m_{n}$ are invariant 
under $f_{\Gamma_{0}}$, then the limit $m_{f}$ is invariant under $f_{\Gamma_{0}}$. As $\bar{m}$ is ergodic, then $m_{f}$ is also ergodic. But by the Ruelle-Perron-Frobenius Theorem (see Theorem 4.1), there is a unique $f_{\Gamma_{0}}$ invariant ergodic probability measure. Thus $m_{f}=\bar{m}$. As $\phi_{t}=\bar{\phi}_{t} \circ \pi_{\Gamma}$, then $m\left(\phi_{t}\right)=\bar{m}\left(\bar{\phi}_{t}\right)$ and

$$
F(t)=\frac{m\left(\phi_{t}\right)}{m\left(\phi_{0}\right)}=\frac{\bar{m}\left(\bar{\phi}_{t}\right)}{\bar{m}\left(\bar{\phi}_{0}\right)}=\frac{m_{f}\left(\bar{\phi}_{t}\right)}{m_{f}\left(\bar{\phi}_{0}\right)}=L_{\mu_{X}}\left(X_{t}\right) .
$$

\section{Positive-definite locus for $G$}

Before we prove the Main Theorem we characterize the zero vectors of $G$ in terms of derivatives of length functions.

Theorem 7.1 Let $v \in T_{X}(\mathrm{QF}(S))$ then $\|v\|_{G}=0$ if and only if for every $\gamma \in \Gamma$, the associated length function $L_{\gamma}: \mathrm{QF}(S) \rightarrow \mathbb{R}$ satisfies

$$
\left(h L_{\gamma}\right)^{\prime}(v)=0 .
$$

Proof We choose our basegroup $\Gamma$ to be the fuchsian group described in Lemma 5.2, ie if $S$ is a genus $g$ surface, then $\Gamma$ is generated by the standard identification of the sides of the regular $4 g$-gon.

We first prove that $\|v\|_{G}=0$ implies that $\left(h L_{\gamma}\right)^{\prime}(v)=0$ for all $\gamma \in \Gamma$. As it is automatically true for $v=0$, we assume that $v \neq 0$ and choose a smooth curve $\alpha:(-\epsilon, \epsilon) \rightarrow \mathrm{QF}(S)$ with $\alpha(0)=X$ and $\alpha^{\prime}(0)=v$ and $\alpha(t)=X_{t}=\left[g_{t}, \Gamma_{t}\right]$ as before. Therefore $g_{t}$ conjugates the action of $\Gamma$ to the action of $\Gamma_{t}$.

We will show that $\left(h L_{\mu}\right)^{\prime}(v)=0$ for all discrete geodesic currents except for the finite set $\mathcal{S}$ considered in Lemma 5.2. Since these are projectively dense in $\mathcal{C}(S)$, and $\left(h L_{\mu}\right)^{\prime}(v)$ is a continuous function that is homogeneous under scaling of currents, it will then follow that $\left(h L_{\mu}\right)^{\prime}(v)=0$ for all geodesic currents.

We note that if $M$ is a loxodromic möbius transformation then the translation distance of $M$ is given by $\log \left|M^{\prime}(z)\right|$ where $z$ is the expanding fixed point of $M$.

Thus if $\gamma \in \Gamma$, with expanding fixed point $z$ then we let $\gamma_{t}=g_{t} \circ \gamma \circ g_{t}^{-1} \in \Gamma_{t}$. Then $\gamma_{t}$ has expanding fixed point $z_{t}=g_{t}(z)$ and

$$
L_{\gamma}\left(X_{t}\right)=\log \left|\gamma_{t}^{\prime}\left(z_{t}\right)\right|
$$

As $\|v\|_{G}=0$, then as $G$ is conformally equivalent to $W,\|v\|_{W}=0$. Therefore by Equation (5), $\operatorname{Var}\left(\dot{\Phi}_{0}, m\right)=0$. But by nondegeneracy of the variance, this gives 
$\dot{\Phi}_{0} \sim 0$ and is a coboundary. Therefore there is a continuous function $u: \Sigma \rightarrow \mathbb{R}$ such that $\dot{\Phi}_{0}(x)=u(\sigma(x))-u(x)$. Iterating we have

$$
\left(S_{n} \dot{\Phi}_{0}\right)(x)=u\left(\sigma^{n}(x)\right)-u(x) .
$$

In particular if $\sigma^{n}(x)=x$ then $\left(S_{n} \dot{\Phi}_{0}\right)(x)=0$.

We consider the set of commensurability classes in $\Gamma$ given by $\mathcal{D}=\{[\gamma] \mid,[\gamma] \notin \mathcal{S}\}$, where $\mathcal{S}$ is the finite set defined in Lemma 5.2. Then the associated set of projective geodesic currents is dense in the space $\mathcal{P} C(S)$ of projective geodesic currents. We now show $\left(h L_{\gamma}\right)^{\prime}(v)=0$ for all $[\gamma] \in \mathcal{D}$.

We let $[\gamma] \in \mathcal{D}$. Then by Lemma 5.2, there is an element $\gamma^{\prime} \in[\gamma]$ whose expanding fixed point $z$ is good and a periodic point of $f_{\Gamma}$. Therefore there is an $n$ such that $f_{\Gamma}^{n}(z)=z$. As $z$ is a good point, we let $x \in \Sigma$ be the unique point such that $\pi_{\Gamma}(x)=z$. As $\pi_{\Gamma} \circ \sigma=f_{\Gamma} \circ \pi_{\Gamma}$, we have

$$
\pi_{\Gamma}\left(\sigma^{n}(x)\right)=f_{\Gamma}^{n}\left(\pi_{\Gamma}(x)\right)=f_{\Gamma}^{n}(z)=z=\pi_{\Gamma}(x) .
$$

As $z$ is a good point, $x$ is the unique preimage of $z$ under $\pi_{\Gamma}$. Therefore $\sigma^{n}(x)=x$.

As $f_{\Gamma}$ is an expanding Markov map and $z$ is a good point, there exists a $\gamma_{z} \in \Gamma$ with expanding fixed point $z$ such that $f_{\Gamma}^{n}=\gamma_{z}$ in an open neighborhood of $z$. Thus elements $\gamma^{\prime}$ and $\gamma_{z}$ have common fixed point $z$. As $\Gamma$ is cocompact, this implies that $\gamma^{\prime}$ and $\gamma_{z}$ are commensurate. Therefore by transitivity of commensurability, $\gamma$ and $\gamma_{z}$ are commensurate.

We let $\gamma_{z, t}=g_{t} \circ \gamma_{z} \circ g_{t}^{-1} \in \Gamma_{t}$. By definition $\pi_{t}(x)=g_{t}\left(\pi_{\Gamma}(x)\right)=g_{t}(z)$ and is therefore the expanding fixed point of $\gamma_{z, t}$. Also as $f_{\Gamma_{t}}=g_{t} \circ f_{\Gamma} \circ g_{t}^{-1}$ we have $f_{\Gamma_{t}}^{n}=g_{t} \circ f_{\Gamma}^{n} \circ g_{t}^{-1}$ and therefore $f_{\Gamma_{t}}^{n}=\gamma_{z, t}$ at $g_{t}(z)$. Thus by the iteration relation in Equation (3) we have

$$
\left(S_{n} \phi_{t}\right)(x)=-\log \left|\left(f_{\Gamma_{t}}^{n}\right)^{\prime}\left(\pi_{t}(x)\right)\right|=-\log \left|\gamma_{z, t}^{\prime}\left(z_{t}\right)\right|=-L_{\gamma_{z}}\left(X_{t}\right) .
$$

Also as $\Phi_{t}(x)=h\left(X_{t}\right) \phi_{t}(x),\left(S_{n} \Phi_{t}\right)(x)=h\left(X_{t}\right)\left(S_{n} \phi_{t}\right)(x)$. Therefore

$$
\left(S_{n} \dot{\Phi}_{0}\right)(x)=\left.\frac{d}{d t}\left(\left(S_{n} \Phi_{t}\right)(x)\right)\right|_{t=0}=\left.\frac{d}{d t}\left(-h\left(X_{t}\right) L_{\gamma_{z}}\left(X_{t}\right)\right)\right|_{t=0}=-\left(h L_{\gamma_{z}}\right)^{\prime}(v) .
$$

As $\left(S_{n} \dot{\Phi}_{0}\right)(x)=0$, we have that $\left(h L_{\gamma_{z}}\right)^{\prime}(v)=0$. Therefore $\left(h L_{\gamma}\right)^{\prime}(v)=0$ for all $[\gamma] \in \mathcal{D}$.

We now prove that if $v$ satisfies $\left(h L_{\gamma}\right)^{\prime}(v)=0$ for all $\gamma \in \Gamma$ then $\|v\|_{G}=0$. As it is true for $v=0$, we assume that $v \neq 0$ and as before, choose a smooth curve $\alpha:(-\epsilon, \epsilon) \rightarrow \mathrm{QF}(S)$ with $\alpha(0)=X$ and $\alpha^{\prime}(0)=v$ and $\alpha(t)=X_{t}=\left[g_{t}, \Gamma_{t}\right]$. 
A Theorem of Livsic that states $f \sim g$ if and only if $\left(S_{n} f\right)(x)=\left(S_{n} g\right)(x)$ whenever $\sigma^{n}(x)=x$ [8]. Therefore we let $\sigma^{n}(x)=x$. Then for $z=\pi_{\Gamma}(x)$ we have $f_{\Gamma}^{n}(z)=z$ and $f_{\Gamma}^{n}=\gamma_{z}$ at $z$ for some $\gamma_{z} \in \Gamma$. As above we have $\left(S_{n} \phi_{t}\right)(x)=-L_{\gamma_{z}}\left(X_{t}\right)$ and

$$
\left(S_{n} \dot{\Phi}_{0}\right)(x)=-\left(h L_{\gamma_{z}}\right)^{\prime}(v) .
$$

By the assumption $\left(h L_{\gamma_{z}}\right)^{\prime}(v)=0$ and therefore $\left(S_{n} \dot{\Phi}_{0}\right)(x)=0$. Therefore by the result of Livsic, $\dot{\Phi}_{0} \sim 0$ and therefore $\operatorname{Var}\left(\dot{\Phi}_{0}, m\right)=0$. It follows that $\|v\|_{W}=0$. As $G$ is conformally equivalent to $W$, then $\|v\|_{G}=0$.

Corollary 7.2 If $\|v\|_{W}=0$ then there is a $k \in \mathbb{R}$ such that

$$
L_{\mu}^{\prime}(v)=k L_{\mu}(X) \text { for all } \mu \in C(S) .
$$

Proof This follows immediately with $k=-h^{\prime}(v) / h(X)$ by applying the product rule to $\left(h L_{\mu}\right)^{\prime}(v)=0$.

We now show that pure bending vectors to the fuchsian locus have zero length.

Lemma 7.3 If $v=J . w$ where $w \in T_{X}(F(S))$ then $\|v\|_{G}=0$.

Proof Let $v=J . w$ where $w \in T_{X}(F(S))$. By Theorem 7.1, to show $\|v\|_{G}=0$ we only need to prove that $\left(h L_{\mu}\right)^{\prime}(v)=0$ for all $\mu \in \mathcal{C}(S)$.

As the complex length functions $\mathcal{L}_{g}$ are holomorphic on $\mathrm{QF}(S)$,

$$
\mathcal{L}_{g}^{\prime}(v)=\mathcal{L}_{g}^{\prime}(J . w)=i \mathcal{L}_{g}^{\prime}(w) .
$$

As $w \in T_{X}(F(S)), \mathcal{L}_{g}^{\prime}(w)$ is real and equal $\mathcal{L}_{g}^{\prime}(w)=L_{g}^{\prime}(w)$. Therefore $\mathcal{L}_{g}^{\prime}(v)=$ $i L_{g}^{\prime}(w)$ is purely imaginary giving

$$
L_{g}^{\prime}(v)=\Re\left(i L_{g}^{\prime}(w)\right)=0 .
$$

Thus $L_{g}^{\prime}(v)=0$ for all $g \in \Gamma$. As $h$ is minimum on the fuchsian locus $F(S)$ then $h^{\prime}(v)=0$ and

$$
\left(h L_{g}\right)^{\prime}(v)=h^{\prime}(v) L_{g}(X)+h(X) L_{g}^{\prime}(v)=0 .
$$

Therefore by Theorem 7.1, $\|v\|_{W}=0$. As $G$ is conformally equivalent to $W$ we therefore have $\|v\|_{G}=0$.

To complete the proof of the Main Theorem, we need to prove the converse of the above Lemma, that pure bending vectors to the fuchsian locus are the only zero length vectors. We do this by showing that the condition that there exists a $k \in \mathbb{R}$ such that $\left(h L_{\mu}\right)^{\prime}(v)=k L_{\mu}(X)$ for all $\mu \in \mathcal{C}(S)$ is sufficient to show that $X$ is fuchsian and 
$v$ is a pure bending vector. This will require a technical analysis of two-generator subgroups of $\Gamma$.

We let $S$ be a closed hyperbolic surface with $S=\mathbb{H}^{2} / \Gamma$ as before and let $g \in \Gamma$. Given any $X=\left[f_{0}, \Gamma_{0}\right] \in \mathrm{QF}(S)$, then $g$ can be identified to a unique element $g\left(\Gamma_{0}\right)=f_{0} \circ g \circ f_{0}^{-1} \in \Gamma_{0} \subseteq \operatorname{PSL}(2, \mathbb{C})$. We can conjugate such that $g\left(\Gamma_{0}\right)$ is of the form

$$
\pm\left(\begin{array}{cc}
\lambda_{g}(X) & 0 \\
0 & \lambda_{g}^{-1}(X)
\end{array}\right) \in \operatorname{PSL}(2, \mathbb{C}), \text { where }\left|\lambda_{g}(X)\right|>1 .
$$

We note that $\lambda_{g}$ is well-defined up to sign and $\lambda_{g}^{2}(X)$ is therefore well-defined.

Therefore the element $g\left(\Gamma_{0}\right)$ is conjugate to the fractional linear map $f(z)=c . z$, where $c=\lambda_{g}^{2}(X)$. Therefore we have that the length function $L_{g}: \mathrm{QF}(S) \rightarrow \mathbb{R}$ is given by $L_{g}(X)=2 \log \left|\lambda_{g}(X)\right|$. Also the holomorphic length function $\mathcal{L}_{g}: \mathrm{QF}(S) \rightarrow \mathbb{C}$ satisfies $L_{g}=\Re\left(\mathcal{L}_{g}\right)$ and $\lambda_{g}^{2}=e^{\mathcal{L}_{g}}$.

Let $X:(-\epsilon, \epsilon) \rightarrow \mathrm{QF}(S)$ be a smooth curve such that $X^{\prime}(0)=v$. We let $X(t)=$ $\left[f_{t}, \Gamma_{t}\right]$. Let $\Gamma_{t}$ be a smooth parameterization. Therefore for $g \in \Gamma_{0}$, the map $\gamma_{g}:(-\epsilon, \epsilon) \rightarrow \operatorname{PSL}(2, \mathbb{C})$ defined by $\gamma_{g}(t)=g\left(\Gamma_{t}\right)$ is a smooth function. Also as $g\left(\Gamma_{t}\right) \in \operatorname{PSL}(2, \mathbb{C})=\operatorname{SL}(2, \mathbb{C}) / \pm I$, we can lift $\gamma_{g}$ to a smooth map $\tilde{\gamma}_{g}:(-\epsilon, \epsilon) \rightarrow$ $\operatorname{SL}(2, \mathbb{C})$.

We then can define $\lambda_{g}:(-\epsilon, \epsilon) \rightarrow \mathbb{C}$ by letting $\lambda_{g}(t)$ equal the largest eigenvalue of $\tilde{\gamma}_{g}(t)$. Furthermore we define the trace functions

$$
t_{g}(t)=\operatorname{tr}\left(\tilde{\gamma}_{g}(t)\right)=\lambda_{g}(t)+\lambda_{g}^{-1}(t) .
$$

Lemma 7.4 Let $v \in T_{X}(\mathrm{QF}(S)), v \neq 0$. If there exists a $k \in \mathbb{R}$ such that

$$
L_{g}^{\prime}(v)=k L_{g}(X) \text { for all } g \in \Gamma
$$

then $\lambda_{g}^{2}$ and $t_{g}^{2}$ are both real and

$$
\Re\left(\frac{\lambda_{g}^{\prime}}{\lambda_{g}}\right)=0
$$

for all $g \in \Gamma$.

Proof As trace functions are holomorphic coordinate function for $\mathrm{QF}(S)$ (see Marden [9]), as $v \neq 0$, there exists $\alpha \in \Gamma$ such that $t_{\alpha}^{\prime}(0) \neq 0$. As

$$
t_{g}^{\prime}=\lambda_{g}^{\prime}-\frac{\lambda_{g}^{\prime}}{\lambda_{g}^{2}}=\lambda_{g}^{\prime}\left(\frac{\lambda_{g}^{2}-1}{\lambda_{g}^{2}}\right)
$$

then $\lambda_{\alpha}^{\prime}(0) \neq 0$. 
As $\Gamma$ is nonelementary, we can choose a $\beta \in \Gamma$ such that $\alpha, \beta$ do not have the same axis. We note that $\alpha, \beta$ have the same axes if and only if there exist $n, m \in \mathbb{Z}$, both nonzero, such that $\alpha^{n}=\beta^{m}$.

By conjugation of $\Gamma_{t}$ we can put $\alpha\left(\Gamma_{t}\right)$ in the diagonal form with

$$
A(t)=\tilde{\gamma}_{\alpha}(t)=\left(\begin{array}{cc}
\lambda_{\alpha}(t) & 0 \\
0 & \lambda_{\alpha}^{-1}(t)
\end{array}\right)
$$

where $\left|\lambda_{\alpha}(t)\right|>1$, and the corresponding matrix for $\beta\left(\Gamma_{t}\right)$ is

$$
B(t)=\tilde{\gamma}_{\beta}(t)=\left(\begin{array}{ll}
a(t) & b(t) \\
c(t) & d(t)
\end{array}\right)
$$

where $a(t) d(t)-b(t) c(t)=1$.

We consider the two-generator subgroup $G_{t}=\langle A(t), B(t)\rangle \subseteq \operatorname{SL}(2, \mathbb{C})$ acting on the upper half space model of $\mathbb{H}^{3}$. Then $A(t)$ fixes $0, \infty$ and preserves the $z$-axis. Since $\alpha$ and $\beta$ have different axes and $\Gamma$ contains no parabolics, it follows that $a(t), d(t)$ are both nonzero.

If $\|v\|_{W}=0$, then by Corollary 7.2, $L_{g}^{\prime}(v)=k L_{g}(X)$ for all $g \in \Gamma$. As $L_{g}=\log \left|\lambda_{g}\right|$, we obtain the equation

$$
\left(\log \left|\lambda_{g}\right|\right)^{\prime}(v)=k \log \left|\lambda_{g}(X)\right| .
$$

As we are only interested in derivatives at 0 for $X(t)$, we will adopt the notation $f^{\prime}=f^{\prime}(0)$.

Therefore for $g=\alpha$ we have $\left(\log \left|\lambda_{\alpha}\right|\right)^{\prime}=k \log \left|\lambda_{\alpha}\right|$ or equivalently

$$
\left(\log \left|\lambda_{\alpha}\right|\right)^{\prime}=\frac{\left|\lambda_{\alpha}\right|^{\prime}}{\left|\lambda_{\alpha}\right|}=k \log \left|\lambda_{\alpha}\right|
$$

Now we consider the element $C_{n}=A^{n} B$ with matrix

$$
C_{n}=\left(\begin{array}{cc}
\lambda_{\alpha}^{n} a & \lambda_{\alpha}^{n} b \\
\lambda_{\alpha}^{-n} c & \lambda_{\alpha}^{-n} d
\end{array}\right)
$$

Let $\mu_{n}, \mu_{n}^{-1}$ be the eigenvalues of $C_{n}$, with $\left|\mu_{n}\right|>1$. Solving for $\mu_{n}$ we have for large $n$ that

$$
\begin{aligned}
\mu_{n} & =\frac{\lambda_{\alpha}^{n} a+\lambda_{\alpha}^{-n} d+\sqrt{\left(\lambda_{\alpha}^{n} a+\lambda_{\alpha}^{-n} d\right)^{2}-4}}{2} \\
& =\lambda_{\alpha}^{n} a\left(1+\lambda_{\alpha}^{-2 n}\left(\frac{a d-1}{a^{2}}\right)+O\left(\lambda_{\alpha}^{-4 n}\right)\right)
\end{aligned}
$$


Let $g_{n}$ be the element of $\Gamma$ corresponding to $C_{n}$; then we have

$$
\begin{aligned}
\log \left|\lambda_{g_{n}}\right| & =n \log \left|\lambda_{\alpha}\right|+\log |a|+\log \left|1+\lambda_{\alpha}^{-2 n}\left(\frac{a d-1}{a^{2}}\right)+O\left(\lambda_{\alpha}^{-4 n}\right)\right| . \\
& =n \log \left|\lambda_{\alpha}\right|+\log |a|+\Re\left(\lambda_{\alpha}^{-2 n}\left(\frac{a d-1}{a^{2}}\right)\right)+O\left(\left|\lambda_{\alpha}\right|^{-4 n}\right) .
\end{aligned}
$$

Then differentiating and using that $\left(\log \left|\lambda_{g_{n}}\right|\right)^{\prime}=k \log \left|\lambda_{g_{n}}\right|$ we obtain

$$
\begin{aligned}
0=n\left(\frac{\left|\lambda_{\alpha}\right|^{\prime}}{\left|\lambda_{\alpha}\right|}-k \log \left|\lambda_{\alpha}\right|\right) & +\left(\frac{|a|^{\prime}}{|a|}-k \log |a|\right)+\Re\left(-2 n \lambda_{\alpha}^{-2 n-1} \lambda_{\alpha}^{\prime}\left(\frac{a d-1}{a^{2}}\right)\right) \\
& +\Re\left(\lambda_{\alpha}^{-2 n}\left(\left(\frac{a d-1}{a^{2}}\right)^{\prime}-k\left(\frac{a d-1}{a^{2}}\right)\right)\right)+O\left(\left|\lambda_{\alpha}\right|^{-4 n}\right) .
\end{aligned}
$$

The first term in the equation above is zero by Equation (9), and the last two go to zero as $n \rightarrow \infty$, so taking the limit gives

$$
\frac{|a|^{\prime}}{|a|}=k \log |a|
$$

Therefore Equation (11) becomes a relation between the real parts of the last two terms, which holds for each $n$. Multiplying by $\left|\lambda_{\alpha}\right|^{2 n} / n$ and taking the limit as $n \rightarrow \infty$ gives

$$
\lim _{n \rightarrow \infty} \Re\left(\left(\frac{\lambda_{\alpha}}{\left|\lambda_{\alpha}\right|}\right)^{-2 n}\left(\frac{\lambda_{\alpha}^{\prime}}{\lambda_{\alpha}}\right)\left(\frac{a d-1}{a^{2}}\right)\right)=0
$$

We let

$$
u=\left(\frac{\lambda_{\alpha}}{\left|\lambda_{\alpha}\right|}\right)^{2}
$$

As we can always choose a sequence $n_{i}$ such that $\lim _{i \rightarrow \infty} u^{-n_{i}}=1$, we have that

$$
\lim _{i \rightarrow \infty} \mathfrak{R}\left(\left(\frac{\lambda_{\alpha}}{\left|\lambda_{\alpha}\right|}\right)^{-2 n_{i}}\left(\frac{\lambda_{\alpha}^{\prime}}{\lambda_{\alpha}}\right)\left(\frac{a d-1}{a^{2}}\right)\right)=\Re\left(\left(\frac{\lambda_{\alpha}^{\prime}}{\lambda_{\alpha}}\right)\left(\frac{a d-1}{a^{2}}\right)\right)=0 .
$$

Therefore we obtain the equation

$$
\Re\left(\left(\frac{\lambda_{\alpha}^{\prime}}{\lambda_{\alpha}}\right)\left(\frac{a d-1}{a^{2}}\right)\right)=0 .
$$

Next we will show that $\lambda_{\alpha}^{2}$ is real; suppose on the contrary that $u=e^{\pi i \theta}$ for $\theta \in$ $(0,2), \theta \neq 1$. We will derive a contradiction from this assumption by considering two cases. 
Case $1 \theta$ is irrational.

If $\theta$ is irrational, then we can choose a sequence $m_{i}$ such that $\lim _{i \rightarrow \infty} u^{-m_{i}}=i$. Then

$$
\lim _{i \rightarrow \infty} \Re\left(\left(\frac{\lambda_{\alpha}}{\left|\lambda_{\alpha}\right|}\right)^{-2 m_{i}}\left(\frac{\lambda_{\alpha}^{\prime}}{\lambda_{\alpha}}\right)\left(\frac{a d-1}{a^{2}}\right)\right)=\Im\left(\left(\frac{\lambda_{\alpha}^{\prime}}{\lambda_{\alpha}}\right)\left(\frac{a d-1}{a^{2}}\right)\right)=0 .
$$

Thus both the real and imaginary parts are zero giving

$$
\left(\frac{\lambda_{\alpha}^{\prime}}{\lambda_{\alpha}}\right)\left(\frac{a d-1}{a^{2}}\right)=0
$$

As $\lambda_{\alpha}^{\prime} \neq 0$ we have $a d=1$. Therefore as $a d-b c=1$, we have $b c=0$ and either $b=0$ or $c=0$. If $b=0$, then $\alpha, \beta$ have common fixed point 0 and if $c=0$, then $\alpha, \beta$ have common fixed point $\infty$. However $\beta$ was chosen so that it does not share any fixed point with $\alpha$, so this is a contradiction.

Case $2 \theta$ is positive rational but not an integer.

We let $\theta=p / q$, where $q>1$ and $p, q$ have no common divisors. Then $u^{q}=1$ and $u^{n q+1}=u$. Then let $n_{i}=i . q-1$. Then $u^{-n_{i}}=u$. Thus

$$
\lim _{i \rightarrow \infty} \Re\left(\left(\frac{\lambda_{\alpha}}{\left|\lambda_{\alpha}\right|}\right)^{-2 n_{i}}\left(\frac{\lambda_{\alpha}^{\prime}}{\lambda_{\alpha}}\right)\left(\frac{a d-1}{a^{2}}\right)\right)=\Re\left(u\left(\frac{\lambda_{\alpha}^{\prime}}{\lambda_{\alpha}}\right)\left(\frac{a d-1}{a^{2}}\right)\right)=0 .
$$

Let $u=x+i y$ where $y \neq 0$. Then

$$
\Re\left(u\left(\frac{\lambda_{\alpha}^{\prime}}{\lambda_{\alpha}}\right)\left(\frac{a d-1}{a^{2}}\right)\right)=x \Re\left(\left(\frac{\lambda_{\alpha}^{\prime}}{\lambda_{\alpha}}\right)\left(\frac{a d-1}{a^{2}}\right)\right)-y \Im\left(\left(\frac{\lambda_{\alpha}^{\prime}}{\lambda_{\alpha}}\right)\left(\frac{a d-1}{a^{2}}\right)\right)=0 .
$$

Therefore by Equation (14), we have

$$
y \Im\left(\left(\frac{\lambda_{\alpha}^{\prime}}{\lambda_{\alpha}}\right)\left(\frac{a d-1}{a^{2}}\right)\right)=0 .
$$

As $y \neq 0$ we obtain the conclusion that both real and imaginary parts are zero giving

$$
\left(\frac{\lambda_{\alpha}^{\prime}}{\lambda_{\alpha}}\right)\left(\frac{a d-1}{a^{2}}\right)=0
$$

This leads to the same contradiction as Case 1 .

Thus we conclude that $\lambda_{\alpha}^{2}$ is real and $\lambda_{\alpha}$ is either purely imaginary or purely real. As $t_{\alpha}=\lambda_{\alpha}+\lambda_{\alpha}^{-1}$, then $t_{\alpha}$ is similarly either purely imaginary or purely real and $t_{\alpha}^{2}$ is real. Therefore we have shown that if $t_{g}^{\prime} \neq 0$ then $t_{g}^{2}$ is real. 
Also as $t_{n}=\lambda_{\alpha}^{n} \cdot a+\lambda^{n} \cdot d$ then

$$
t_{n}^{\prime}=n \lambda_{\alpha}^{n-1} \lambda_{\alpha}^{\prime} a+\lambda_{\alpha}^{n} a^{\prime}-n \lambda_{\alpha}^{-n-1} \lambda_{\alpha}^{\prime} a+\lambda_{\alpha}^{-n} a^{\prime} .
$$

Thus

$$
\lim _{n \rightarrow \infty}\left(\frac{t_{n}^{\prime}}{n \lambda_{\alpha}^{n}}\right)=\frac{\lambda_{\alpha}^{\prime}}{\lambda_{\alpha}} a
$$

and therefore for large $n, t_{n}^{\prime} \neq 0$. Choose $n_{0}$ such that $t_{n}^{\prime} \neq 0$ for $n>n_{0}$.

We let $n>n_{0}$. By the above, $t_{n}^{2}$ is real and

$$
t_{n}^{2}=\left(\lambda_{\alpha}^{n} a+\lambda_{\alpha}^{-n} d\right)^{2}=\lambda_{\alpha}^{2 n} a^{2}+2 a d+\lambda_{\alpha}^{-2 n} d^{2} .
$$

As $t_{n}^{2}$ is real and $\lambda_{\alpha}^{2}$ is real, we have

$$
\Im\left(t_{n}^{2}\right)=0=\lambda_{\alpha}^{2 n} \Im\left(a^{2}\right)+2 \Im(a d)+\lambda_{\alpha}^{-2 n} \Im\left(d^{2}\right) .
$$

Taking limits we have

Therefore

$$
\lim _{n \rightarrow \infty} \frac{\Im\left(t_{n}^{2}\right)}{\lambda_{\alpha}^{2 n}}=\Im\left(a^{2}\right)=0 .
$$

and finally

$$
\lim _{n \rightarrow \infty} \Im\left(t_{n}^{2}\right)=2 \Im(a d)=0
$$

$$
\lim _{n \rightarrow \infty}\left(\lambda_{\alpha}^{2 n} \Im\left(t_{n}^{2}\right)\right)=\Im\left(d^{2}\right)=0 .
$$

Thus $a^{2}, d^{2}, a d$ are all real. Applying this to Equation (14) we have

$$
\Re\left(\left(\frac{\lambda_{\alpha}^{\prime}}{\lambda_{\alpha}}\right)\left(\frac{a d-1}{a^{2}}\right)\right)=\left(\frac{a d-1}{a^{2}}\right) \Re\left(\frac{\lambda_{\alpha}^{\prime}}{\lambda_{\alpha}}\right)=0 .
$$

Therefore we have

$$
\Re\left(\frac{\lambda_{\alpha}^{\prime}}{\lambda_{\alpha}}\right)=0
$$

As the only assumption on $\alpha$ was that $t_{\alpha}^{\prime}$ and therefore $\lambda_{\alpha}^{\prime}$ was nonzero, we have

$$
\Re\left(\frac{\lambda_{g}^{\prime}}{\lambda_{g}}\right)=0 \text { for all } g \in \Gamma .
$$

Also as $t_{\beta}^{2}=(a+d)^{2}=a^{2}+2 a d+d^{2}$, then we have that $t_{\beta}^{2}$ is real. As $\beta$ was arbitrarily chosen, we therefore have that $t_{g}^{2}$ is real for all $g \in \Gamma$. As $t_{g}^{2}$ is real, then $\lambda_{g}^{2}$ is also real.

Lemma 7.5 If $v \in T_{X}(\mathrm{QF}(S))$ and there exists a $k \in \mathbb{R}$ such that $L_{g}^{\prime}(v)=k . L_{g}(X)$ then $k=0$. 
Proof If $v=0$ then $L_{g}^{\prime}(v)=0$ and obviously $k=0$. Therefore we assume $v \neq 0$. Let $g \in \Gamma$, and $\lambda_{g}=\left|\lambda_{g}\right| e^{i \theta}$. Then

$$
\frac{\lambda_{g}^{\prime}}{\lambda_{g}}=\frac{\left|\lambda_{g}\right|^{\prime} e^{i \theta}+\left|\lambda_{g}\right| e^{i \theta} i \theta^{\prime}}{\left|\lambda_{g}\right| e^{i \theta}}=\frac{\left|\lambda_{g}\right|^{\prime}}{\left|\lambda_{g}\right|}+i \theta^{\prime} .
$$

Then by Equation (9) and Lemma 7.4,

$$
0=\Re\left(\frac{\lambda_{g}^{\prime}}{\lambda_{g}}\right)=\frac{\left|\lambda_{g}\right|^{\prime}}{\left|\lambda_{g}\right|}=k \log \left|\lambda_{g}\right|
$$

Thus we have $k \cdot \log \left|\lambda_{g}\right|=0$. As $\left|\lambda_{g}\right|>1, \log \left|\lambda_{g}\right| \neq 0$ and therefore $k=0$.

We now are ready to prove the Main Theorem.

Proof of Main Theorem As Lemma 7.3 proves one direction of the Main Theorem, we only need to prove if $v \in T_{X}(\mathrm{QF}(S)), v \neq 0$, and $\|v\|_{G}=0$ then $X \in F(S)$ and $v=J . w$ for some $w \in T_{X}(F(S))$.

Let $v \in T_{X}(\mathrm{QF}(S)), v \neq 0$, and $\|v\|_{G}=0$. As $W$ is a multiple of $G,\|v\|_{W}=0$. Then by Corollary 7.2, there is a $k \in \mathbb{R}$ such that $L_{g}^{\prime}(v)=k L_{g}(X)$ for all $g \in \Gamma$. Then by Lemma 7.5, $k=0$ giving $L_{g}^{\prime}(v)=0$ for all $g \in \Gamma$.

We pick $\alpha, \beta$ as in Lemma 7.4. For the group $G_{0}=\langle A(0), B(0)\rangle$ we have $t_{\alpha}^{2}, \lambda_{\alpha}^{2}$, $a^{2}, a d, d^{2}$ are all real. Therefore the fractional linear map given by $A$ is $f_{A}(z)=$ $\lambda_{\alpha}^{2} . z \in \operatorname{PSL}(2, \mathbb{R})$.

As $a d-b c=1$, we therefore have that $b c=a d-1$ is real. Therefore $b=r e^{i \theta}$ and $c=s e^{-i \theta}$ where $r, s$ are real.

If $a, d$ are both real, we conjugate $G_{0}$ by rotation $R$ about the axis of $A$ by angle $\theta$. Then as $R, A$ commute, $R A R^{-1}=A$ and

$$
R B R^{-1}=\left(\begin{array}{cc}
e^{-i \theta / 2} & 0 \\
0 & e^{i \theta / 2}
\end{array}\right)\left(\begin{array}{ll}
a & b \\
c & d
\end{array}\right)\left(\begin{array}{cc}
e^{i \theta / 2} & 0 \\
0 & e^{-i \theta / 2}
\end{array}\right)=\left(\begin{array}{ll}
a & r \\
s & d
\end{array}\right) .
$$

Therefore the fractional linear map given by $R B R^{-1}$ is in $\operatorname{PSL}(2, \mathbb{R})$.

If $a, d$ are both imaginary, we conjugate by a rotation $R$ about the axis of $A$ by angle $\pi+\theta$. Then

$$
R B R^{-1}=\left(\begin{array}{cc}
a & i r \\
i s & d
\end{array}\right)
$$

Thus as each entry is imaginary, the fractional linear map is in $\operatorname{PSL}(2, \mathbb{R})$.

Therefore we have conjugated $G_{0}$ to a subgroup of $\operatorname{PSL}(2, \mathbb{R})$. Thus $G_{0}$ has limit set contained in a Euclidean line $L_{0}$ through the origin and $G_{0}$ preserves a hyperbolic 
plane $H_{0}$ containing the axis of $A$. We conclude that if $\alpha \in \Gamma_{0}$ has $\lambda_{\alpha}^{\prime} \neq 0$ then for any $\beta \in \Gamma_{0}$, the axes of $\alpha$ and $\beta$ are contained in the same geometric circle.

If $X \notin F(S)$ then there is an element $\gamma \in \Gamma$ such that the associated fractional linear map $C \in \Gamma_{0}$ does not preserve $H_{0}$. Then we have as before that the group $G_{1}=\langle A, C\rangle$ can be conjugated to a subgroup of $\operatorname{PSL}(2, \mathbb{R})$. Therefore $\Gamma_{1}$ preserves a line $L_{1}$, and hyperbolic plane $H_{1}$ containing the axis of $A$. As by assumption $C$ does not preserve $H_{0}$ then $H_{0} \neq H_{1}$ and therefore $L_{1} \neq L_{0}$. Thus $L_{1} \cap L_{0}=\{0, \infty\}$. By conjugation, we assume that $L_{0}$ is the real axis.

We note that if $g, h$ are loxodromic hyperbolic elements, then the axis of $g h g^{-1}$ is the image of the axis of $h$ under $g$.

Thus we conjugate $\alpha$ by $\beta \alpha^{n}$ to get

$$
\alpha_{n}=\left(\beta \alpha^{n}\right)^{-1} \alpha\left(\beta \alpha^{n}\right)=\alpha^{-n}\left(\beta^{-1} \alpha \beta\right) \alpha^{n} .
$$

Then as $\alpha_{n}$ is a conjugate of $\alpha$ we have $\lambda_{\alpha_{n}}^{\prime}=\lambda_{\alpha}^{\prime} \neq 0$. Let $\left.A_{n}=\left(B A^{n}\right)^{-1}\right) A\left(B A^{n}\right)=$ $A^{-n}\left(B^{-1} A B\right) A^{n} \in \Gamma_{0}$. Then the endpoints of the axes of $A_{n}$ and $C$ must be contained in a geometric circle. Also the axis of $A_{n}$ is the image of the axis of $B A B^{-1}$ under $A^{-n}$. Therefore we let $a, b$ be the endpoints of the axis of $B^{-1} A B$. As $A, B$ are noncommensurate, their axes do not have common endpoints. Therefore $a, b \in \mathbb{R}$ and are not equal 0 or $\infty$. Then the endpoint of the axis of $A_{n}$ are $a_{n}, b_{n}$ where $a_{n}=A^{-n}(a)=\lambda_{\alpha}^{-2 n} a, b_{n}=A^{-n}(b)=\lambda_{\alpha}^{-2 n} b$.

Let $z, w \in L_{1}$ be the endpoints of the axis of $C$. As $L_{1}$ is not the real axis, then $z=r e^{i \theta}, w=s e^{i \theta}$ where $r, s \in \mathbb{R}$, and $e^{i \theta}$ is not real. As the axes of $C$ and $A_{n}$ are on the same geometric circle, the cross ratio $\left(a_{n}, z ; b_{n}, w\right)$ is real for all $\mathrm{n}$ :

$$
\left(a_{n}, z ; b_{n}, w\right)=\frac{\left(a_{n}-b_{n}\right)(z-w)}{\left(a_{n}-w\right)\left(z-b_{n}\right)}=\frac{\lambda_{\alpha}^{-2 n}(a-b)(r-s) e^{i \theta}}{\left(\lambda_{\alpha}^{-2 n} a-s e^{i \theta}\right)\left(r e^{i \theta}-\lambda_{\alpha}^{-2 n} b\right)}
$$

Therefore as $\Im\left(a_{n}, z ; b_{n}, w\right)=0$ and $\lambda_{\alpha}^{2}$ is real then

$$
\begin{aligned}
0=\lim _{n \rightarrow \infty} \lambda_{\alpha}^{2 n} . \Im\left(a_{n}, z ; b_{n}, w\right) & =\lim _{n \rightarrow \infty} \Im\left(\frac{(a-b)(r-s) e^{i \theta}}{\left(\lambda_{\alpha}^{-2 n} a-s e^{i \theta}\right)\left(r e^{i \theta}-\lambda \bar{\alpha}^{-2 n} b\right)}\right) \\
& =\Im\left(\frac{(a-b)(r-s) e^{i \theta}}{-s r e^{2 i \theta}}\right)=\frac{(a-b)(r-s)}{-r s} \Im\left(e^{-i \theta}\right) .
\end{aligned}
$$

Thus $\Im\left(e^{-i \theta}\right)=0$, and therefore $e^{i \theta}$ is real. But by assumption $e^{i \theta}$ is not real, which gives us our contradiction. Thus $X \in F(S)$.

Finally as $X \in F(S)$, we have the decomposition [6]

$$
T_{X}(\mathrm{QF}(S))=T_{X}(F(S)) \oplus J\left(T_{X}(F(S))\right) .
$$


If $v \in T_{X}(F(S))$ then $\mathcal{L}_{\alpha}^{\prime}(v)=L_{\alpha}^{\prime}(v)$ and is real. Therefore if $v \in T_{X}(\mathrm{QF}(S))$, then $v=v_{1}+J . v_{2}$ where $v_{i} \in T_{X}(F(S))$. Therefore

$$
\begin{aligned}
L_{g}^{\prime}(v)=\Re\left(\mathcal{L}_{g}^{\prime}(v)\right) & =\Re\left(\mathcal{L}_{g}^{\prime}\left(v_{1}\right)+\mathcal{L}_{g}^{\prime}\left(J . v_{2}\right)\right) \\
& =\Re\left(\mathcal{L}_{g}^{\prime}\left(v_{1}\right)+i \mathcal{L}_{g}^{\prime}\left(v_{2}\right)\right)=\Re\left(L_{g}^{\prime}\left(v_{1}\right)+i L_{g}^{\prime}\left(v_{2}\right)\right)=L_{g}^{\prime}\left(v_{1}\right) .
\end{aligned}
$$

Therefore if $L_{g}^{\prime}(v)=0$ for all $g \in \Gamma$, then $L_{g}^{\prime}(v)=L_{g}^{\prime}\left(v_{1}\right)=0$ for all $g \in \Gamma$. But this implies that $v_{1}=0$ and therefore $v=J . v_{2}$. Thus $X \in F(S)$ and $v=J . w$ for some $w \in T_{X}(F(S))$.

\section{Critical points of Hausdorff dimension}

We will now use the description of the positive definite locus of $G$ to obtain information about the critical points of $h: \mathrm{QF}(S) \rightarrow \mathbb{R}$.

If $f: X \rightarrow \mathbb{R}$ is a smooth map, then $x \in X$ is a critical point of the differential $f^{\prime}(x): T_{x}(X) \rightarrow \mathbb{R}$ is the trivial linear function.

If $x$ is a critical point of $f$ then the Hessian of $f$ is a well-defined two-form which we label $f^{\prime \prime}(x)$. Then we can decompose $T_{x}(X)=V_{+} \oplus V_{0} \oplus V_{-}$where the subspaces are mutually orthogonal and $f^{\prime \prime}(x)$ is positive-definite (respectively, zero, negativedefinite) on $V_{+}$(resp. $V_{0}, V_{-}$). The positive definite (respectively zero, negativedefinite) dimension of $f^{\prime \prime}(x)$ is the dimension of $V_{+}$(resp. $V_{0}, V_{-}$).

As $h \geq 1$ and $h=1$ on the fuchsian subspace $F(S)$ it follows that each $h$ is minimum (and therefore critical) at each point of $F(S)$. Thus for $X \in F(S), h^{\prime \prime}(X)$ has negative definite dimension zero and trivial dimension at least $\operatorname{dim}(F(S))=6 g-6$. In [6], we show that $h^{\prime \prime}(X)$ has positive definite dimension $6 g-6$. We generalize this to all critical points of $h$ to prove Theorem 1.2.

Theorem 1.2 If $X \in \mathrm{QF}(S)$ is a critical point of $h: \mathrm{QF}(S) \rightarrow \mathbb{R}$ then $X$ has positive definite dimension at least $6 g-6$. In particular $h$ has no local maxima.

Proof As the Theorem is true for $X \in F(S)$ [6], we assume that $X \notin F(S)$. By [5], if $\mu_{X}$ is the unit Patterson-Sullivan geodesic current for $X \in \mathrm{QF}(S)$ then the real valued function $Y \rightarrow h(Y) L_{\mu_{X}}(Y)$ on $\mathrm{QF}(S)$ is minimum at $X$. Therefore $\left(h L_{\mu_{X}}\right)^{\prime}(X)=0$.

If $X$ is a critical point of $h$ then $h^{\prime}(X)=0$ and therefore by the product rule

$$
h^{\prime}(X) L_{\mu_{X}}(X)+h(X) L_{\mu_{X}}^{\prime}(X)=h(X) L_{\mu_{X}}^{\prime}(X)=0 .
$$


As $h(X) \neq 0$ then $L_{\mu_{X}}^{\prime}(X)=0$ and therefore $L_{\mu_{X}}$ has a critical point at $X$. We note that the holomorphic length function $\mathcal{L}_{\mu_{X}}$ satisfies

$$
\mathfrak{R}\left(\mathcal{L}_{\mu_{X}}\right)=L_{\mu_{X}} .
$$

Therefore as $L_{\mu_{X}}^{\prime}(X)=0$, then for all $v \in T_{X}(\mathrm{QF}(S))$,

$$
\Re\left(\mathcal{L}_{\mu_{X}}^{\prime}(v)\right)=L_{\mu_{X}}^{\prime}(v)=0 .
$$

Therefore applying this to $J . v$ we have

$$
0=\mathfrak{R}\left(\mathcal{L}_{\mu_{X}}^{\prime}(J . v)\right)=\mathfrak{R}\left(i \mathcal{L}_{\mu_{X}}^{\prime}(v)\right)=-\Im\left(\mathcal{L}_{\mu_{X}}^{\prime}(v)\right) .
$$

Thus $\mathcal{L}_{\mu_{X}}^{\prime}(v)$ has real and imaginary part zero and therefore $\mathcal{L}_{\mu_{X}}^{\prime}(v)=0$ for all $v \in T_{X}(\mathrm{QF}(S))$. Thus $\mathcal{L}_{\mu_{X}}^{\prime}(X)=0$ and we have a well-defined complex bilinear 2 -form $\mathcal{L}_{\mu_{X}}^{\prime \prime}(X)$.

As the two-form $G_{X}$ is given by $G_{X}=\left(h L_{\mu_{X}}\right)^{\prime \prime}(X)$ and both $h, L_{\mu_{X}}$ are critical at $X$ we have

$$
G_{X}=h^{\prime \prime}(X) L_{\mu}(X)+2 h^{\prime}(X) L_{\mu_{X}}^{\prime}(X)+h(X) L_{\mu}^{\prime \prime}(X)=h^{\prime \prime}(X)+h(X) L_{\mu_{X}}^{\prime \prime}(X) .
$$

We write $T_{X}(\mathrm{QF}(S))=V_{+} \oplus V_{0} \oplus V_{-}$where $L_{\mu_{X}}^{\prime \prime}$ is positive-definite (respectively, zero, negative-definite) on $V_{+}$(resp. $\left.V_{0}, V_{-}\right)$. Since $L_{\mu_{X}}^{\prime \prime}$ is the real part of a complex bilinear form, the complex structure $J$ is an isomorphism from $V_{+}$to $V_{-}$, and each of these has dimension at most $(6 g-6)$. Therefore $\operatorname{dim}\left(V_{-} \oplus V_{0}\right) \geq 6 g-6$.

As $X \notin F(S)$ then $G_{X}$ is positive definite. As $G_{X}=h^{\prime \prime}(X)+h(X) L_{\mu_{X}}^{\prime \prime}(X)$, then $h^{\prime \prime}(X)$ must be positive-definite on $V_{0} \oplus V_{-}$. Therefore $h$ has positive definite dimension at least $6 g-6$ at $X$.

We now give a proof of McMullen's result (Theorem 1.4) that the Weil-Petersson and Hausdorff norms are equal on $J(T F(S))$.

Proof If $w=J . v$ for $v \in T_{X}(F(S))$ then by the Main Theorem we have $\|w\|_{G}=0$. As $h(X)=1$, and by holomorphicity, $L_{\mu_{X}}^{\prime \prime}(J . v, J . v)=-L_{\mu_{X}}^{\prime \prime}(v)$ we have

$$
0=\|w\|_{G}^{2}=h^{\prime \prime}(J . v, J . v)+h(X) . L_{\mu_{X}}^{\prime \prime}(J . v, J . v)=\|v\|_{H}^{2}-L_{\mu_{X}}^{\prime \prime}(v, v) .
$$

Thus

$$
\|v\|_{H}^{2}=L_{\mu_{X}}^{\prime \prime}(v, v) .
$$

In [19], Wolpert showed that $L_{\mu_{X}}^{\prime \prime}(v, v)=\|v\|_{g}$ and the Theorem follows. 


\section{References}

[1] L Bers, Simultaneous uniformization, Bull. Amer. Math. Soc. 66 (1960) 94-97 MR0111834

[2] F Bonahon, Shearing hyperbolic surfaces, bending pleated surfaces and Thurston's symplectic form, Ann. Fac. Sci. Toulouse Math. (6) 5 (1996) 233-297 MR1413855

[3] R Bowen, Periodic orbits for hyperbolic flows, Amer. J. Math. 94 (1972) 1-30 MR0298700

[4] R Bowen, Hausdorff dimension of quasicircles, Inst. Hautes Études Sci. Publ. Math. (1979) 11-25 MR556580

[5] M Bridgeman, E C Taylor, Patterson-Sullivan measures and quasi-conformal deformations, Comm. Anal. Geom. 13 (2005) 561-589 MR2171692

[6] M Bridgeman, E C Taylor, An extension of the Weil-Petersson metric to quasiFuchsian space, Math. Ann. 341 (2008) 927-943 MR2407332

[7] S P Kerckhoff, Earthquakes are analytic, Comment. Math. Helv. 60 (1985) 17-30 MR787659

[8] A Livsic, Cohomology properties of dynamical systems, Math. USSR-Izv. 6 (1972)

[9] A Marden, The geometry of finitely generated kleinian groups, Ann. of Math. (2) 99 (1974) 383-462 MR0349992

[10] B Maskit, Kleinian groups, Grund. der Math. Wissenschaften 287, Springer, Berlin (1988) MR959135

[11] C T McMullen, Thermodynamics, dimension and the Weil-Petersson metric, Invent. Math. 173 (2008) 365-425 MR2415311

[12] P J Nicholls, The ergodic theory of discrete groups, London Math. Society Lecture Note Ser. 143, Cambridge Univ. Press (1989) MR1041575

[13] W Parry, M Pollicott, Zeta functions and the periodic orbit structure of hyperbolic dynamics, Astérisque 187-188 (1990) MR1085356

[14] D Ruelle, Repellers for real analytic maps, Ergodic Theory Dynamical Systems 2 (1982) 99-107 MR684247

[15] K Sigmund, On dynamical systems with the specification property, Trans. Amer. Math. Soc. 190 (1974) 285-299 MR0352411

[16] D Sullivan, The density at infinity of a discrete group of hyperbolic motions, Inst. Hautes Études Sci. Publ. Math. (1979) 171-202 MR556586

[17] D Sullivan, Entropy, Hausdorff measures old and new, and limit sets of geometrically finite Kleinian groups, Acta Math. 153 (1984) 259-277 MR766265

[18] W P Thurston, The geometry and topology of three-manifolds, Princeton Univ. Math. Dept. Lecture Notes (1979) Available at http://msri.org/publications/books/ gt3m/ 
[19] S A Wolpert, Thurston's Riemannian metric for Teichmüller space, J. Differential Geom. 23 (1986) 143-174 MR845703

Department of Mathematics, Boston College

Chestnut Hill, MA 02167

bridgem@bc.edu

http://www2.bc.edu/ bridgem/

Proposed: Shigeyuki Morita

Received: 9 February 2009

Seconded: Benson Farb, Martin Bridson

Revised: 3 January 2010 